\title{
Establishing an Electrostatics Paradigm for Membrane Electroporation in the Framework of Dissipative Particle Dynamics
}

\author{
Supplementary Information
}

Rakesh Vaiwala, Sameer Jadhav, and Rochish Thaokar

Department of Chemical Engineering,

Indian Institute of Technology Bombay, Mumbai-400 076, India.
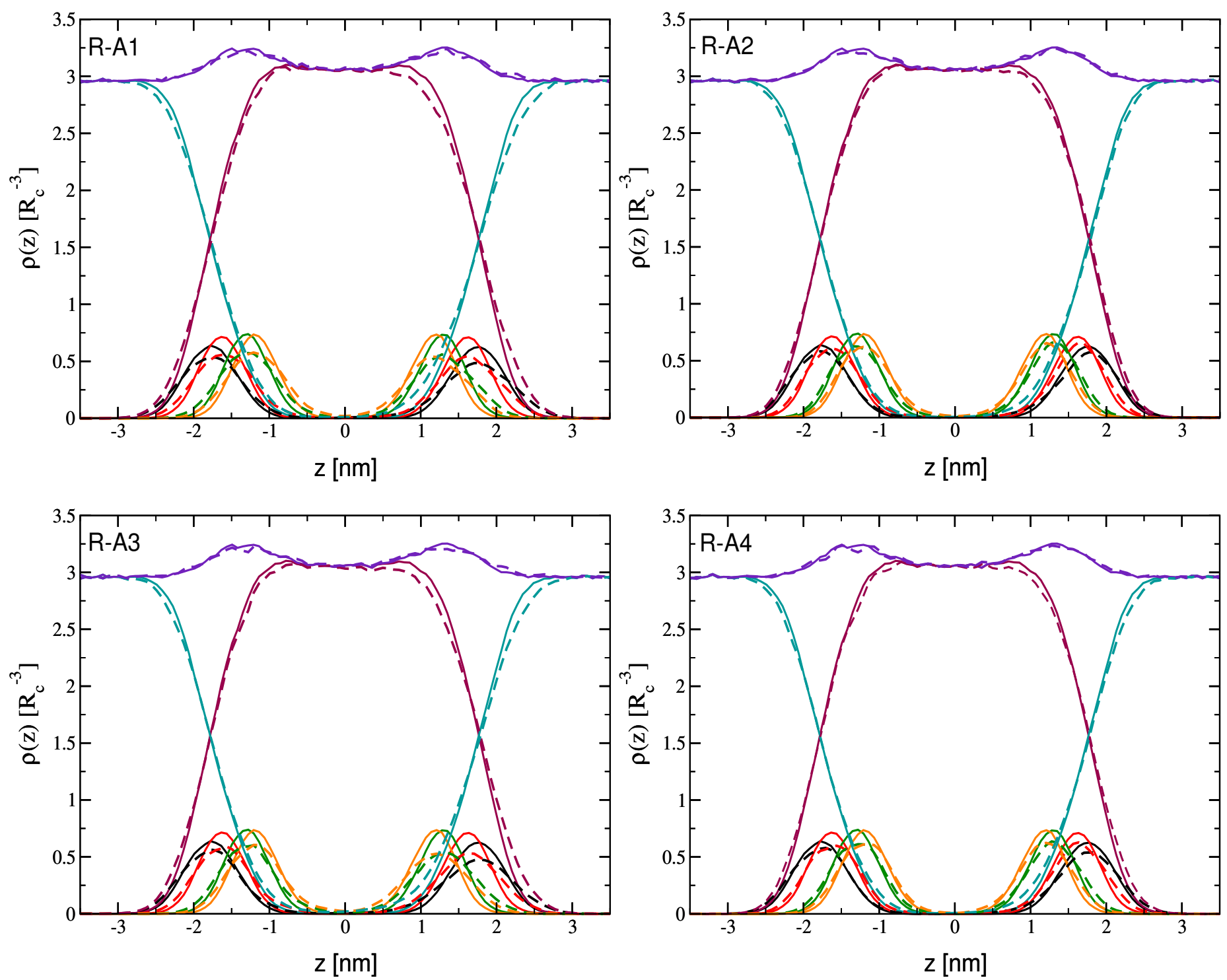

SI. Figure 1 Bead density profiles for four independent simulation runs R-A1, R-A2, R-A3 and R-A4, averaged over simulation time. For comparison, solid lines correspond to density profiles under no applied electric field, while dotted lines refer to the density distributions at applied field $E_{z}=-1.8$ $k_{B} T / e R_{C}$. Black color indicates choline, red phosphate, green carbonyl-1, orange carbonyl-2, brown lipid, cyan water, and violet total density. 

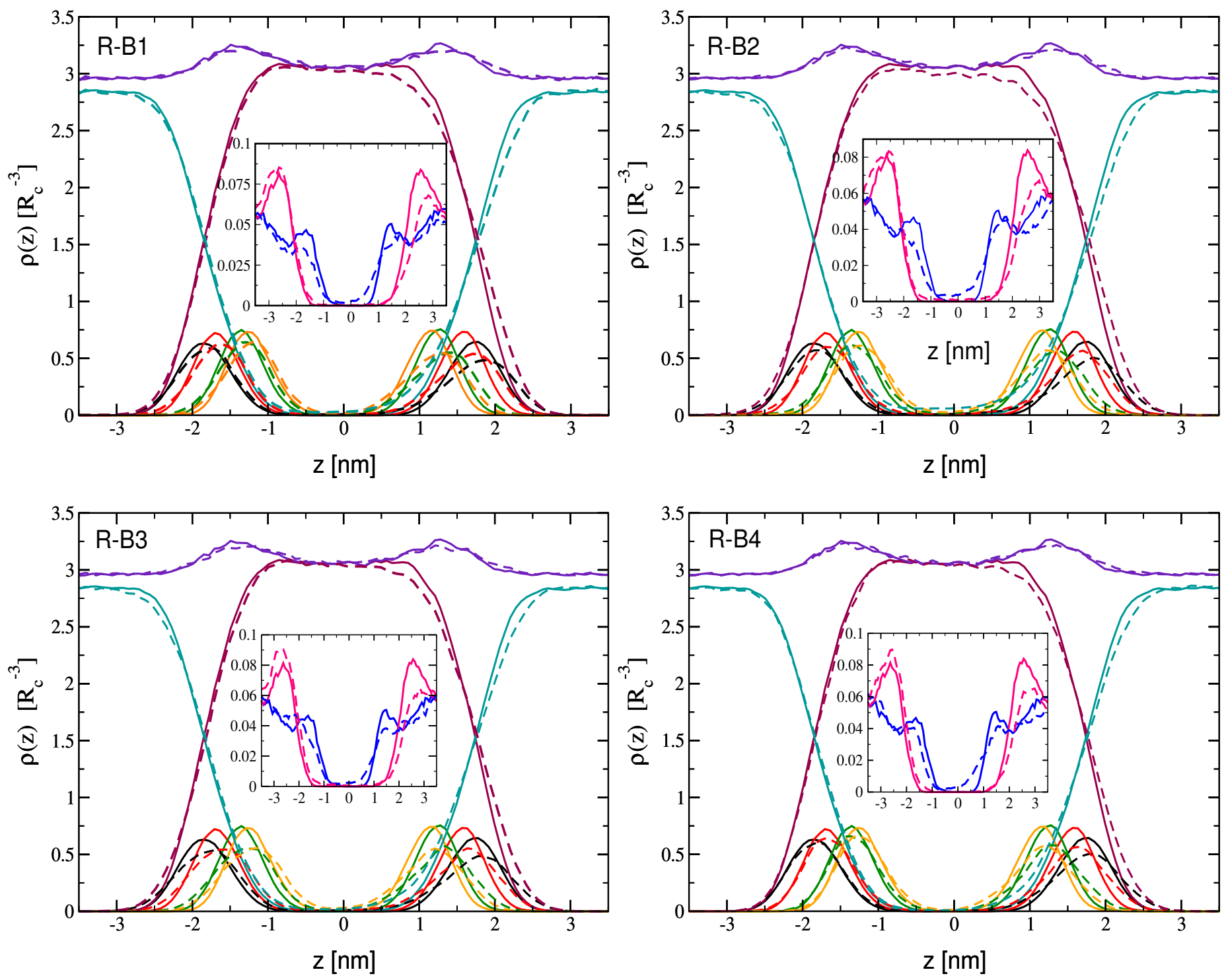

SI. Figure 2 Bead density profiles for simulation runs R-B1, R-B2, R-B3 and R-B4, averaged over simulation time. For comparison, solid lines correspond to density profiles under no applied electric field, while dotted lines refer to the density distributions at applied field $E_{z}=-1.4 k_{B} T / e R_{c}$. Black color indicates choline, red phosphate, green carbonyl-1, orange carbonyl-2, brown lipid, cyan water, and violet total density. The inset depicts ion concentrations for (blue) cation and (pink) anion. 

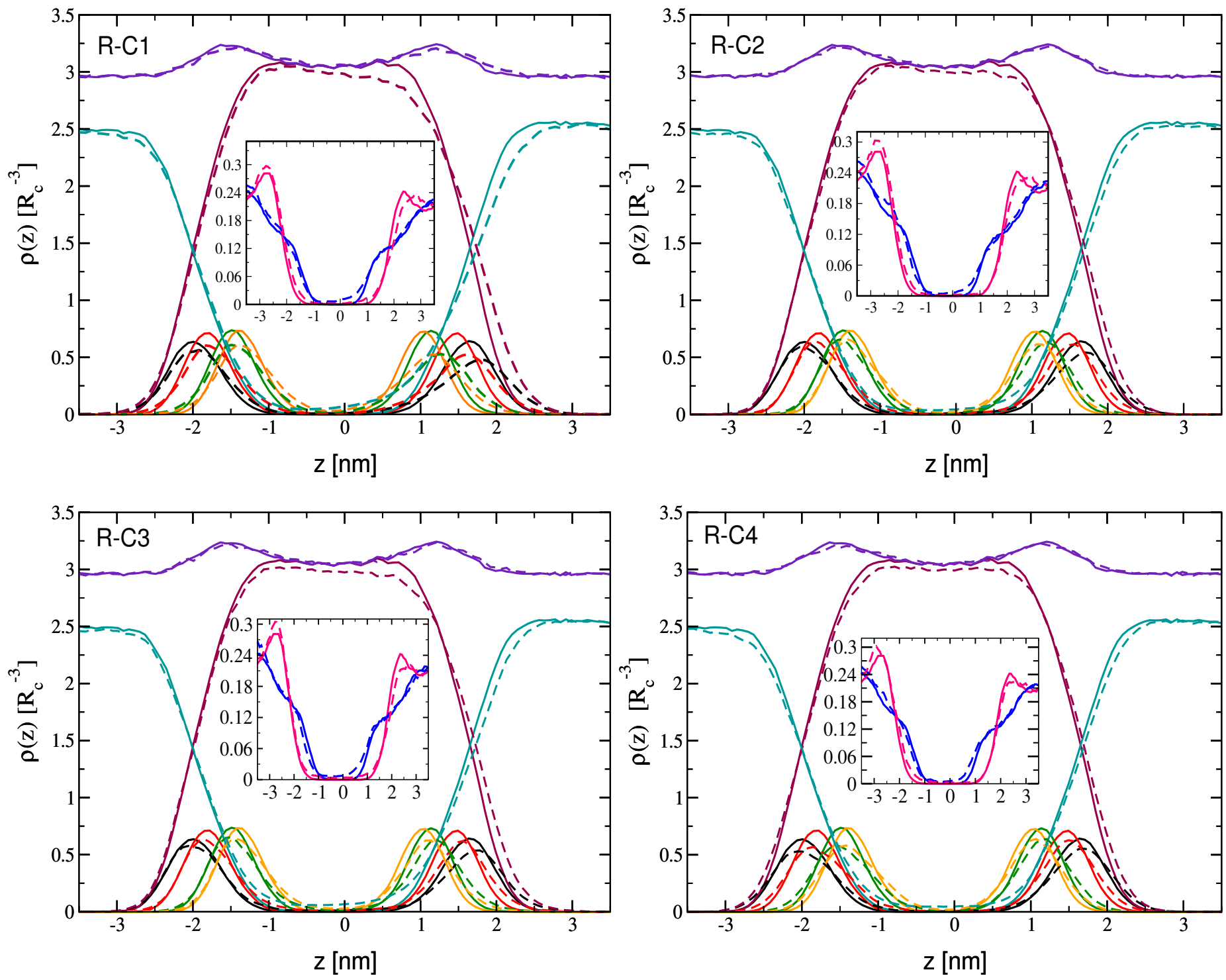

SI. Figure 3 Bead density profiles for simulation runs R-C1, R-C2, R-C3 and R-C4, averaged over simulation time. For comparison, solid lines correspond to density profiles under no applied electric field, while dotted lines refer to the density distributions at applied field $E_{z}=-1.4 k_{B} T / e R_{c}$. Black color indicates choline, red phosphate, green carbonyl-1, orange carbonyl-2, brown lipid, cyan water, and violet total density. The inset depicts ion concentrations for (blue) cation and (pink) anion. 

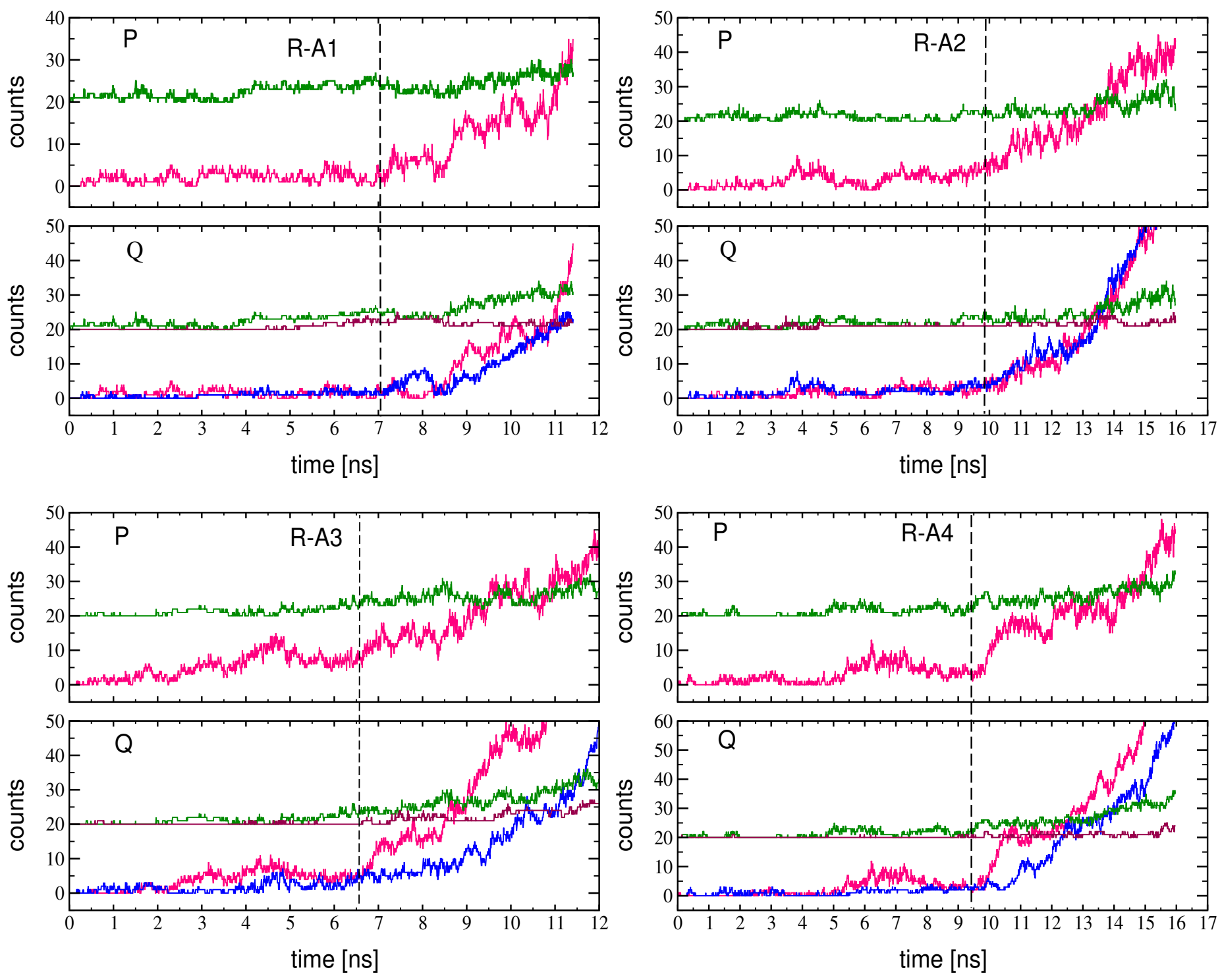

SI. Figure 4 Species flux through a pore from four repeated simulations R-A1, R-A2, R-A3 and R-A4 showing (P) number of water (pink) and choline (green) in the core of bilayer, and (Q) cumulative flow of water and choline into the core through anodic (pink and green) and cathodic (blue and brown) interfaces. The counts for the choline are shifted by 20 for visual clarity. The vertical dotted line indicates onset of pre-pore creation. 

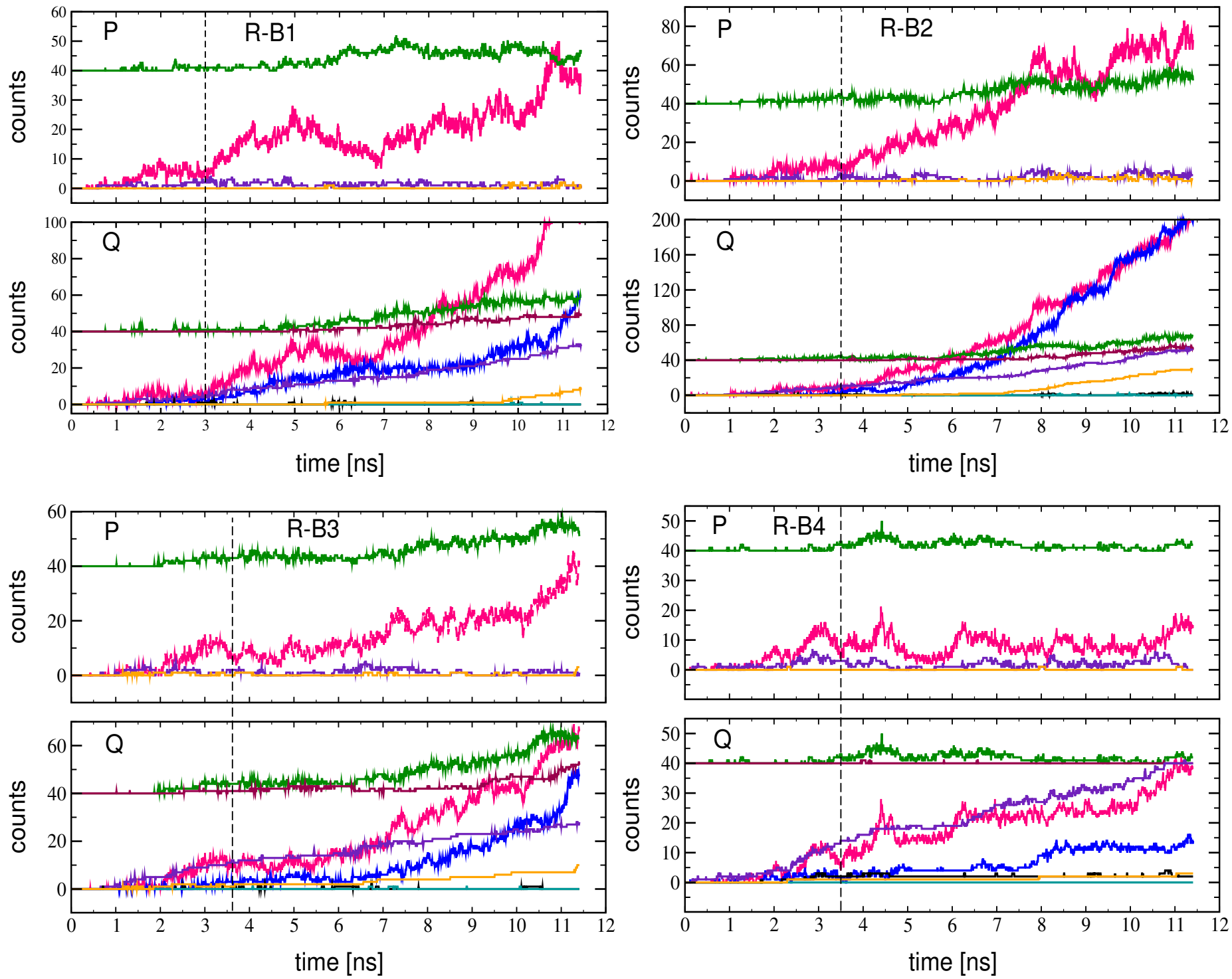

SI. Figure 5 Species flux through a pore from simulations R-B1, R-B2, R-B3 and R-B4 showing (P) number of water (pink), choline (green), cation (violet) and anion (orange) in the core of bilayer, and (Q) cumulative flow of water and choline into the core through anodic (pink and green) and cathodic (blue and brown) interfaces. Also shown in $(\mathrm{Q})$ are the cumulative fluxes for cation (violet and black) and anion (cyan and orange) through anodic (violet and cyan) and cathodic (black and orange) interfaces. The counts for choline are shifted by 40 . The vertical dotted line indicates the time when a pre-pore forms. 

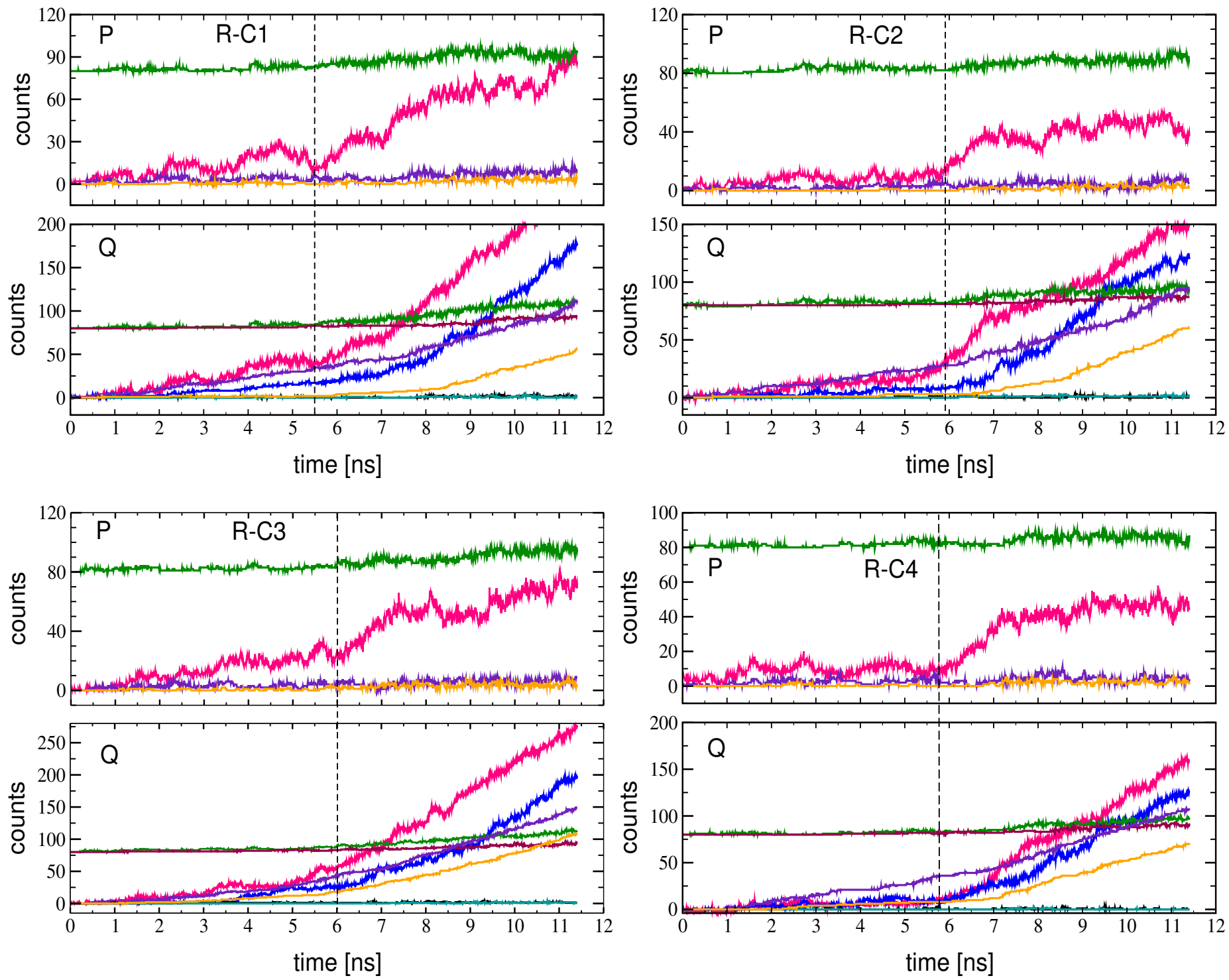

SI. Figure 6 Species flux through a pore from simulations R-C1, R-C2, R-C3 and R-C4 showing (P) number of water (pink), choline (green), cation (violet) and anion (orange) in the core of bilayer, and (Q) cumulative flow of water and choline into the core through anodic (pink and green) and cathodic (blue and brown) interfaces. Also shown in (Q) are the cumulative fluxes for cation (violet and black) and anion (cyan and orange) through anodic (violet and cyan) and cathodic (black and orange) interfaces. The counts for choline are shifted by 80 . The vertical dotted line indicates the time when a pre-pore forms. 

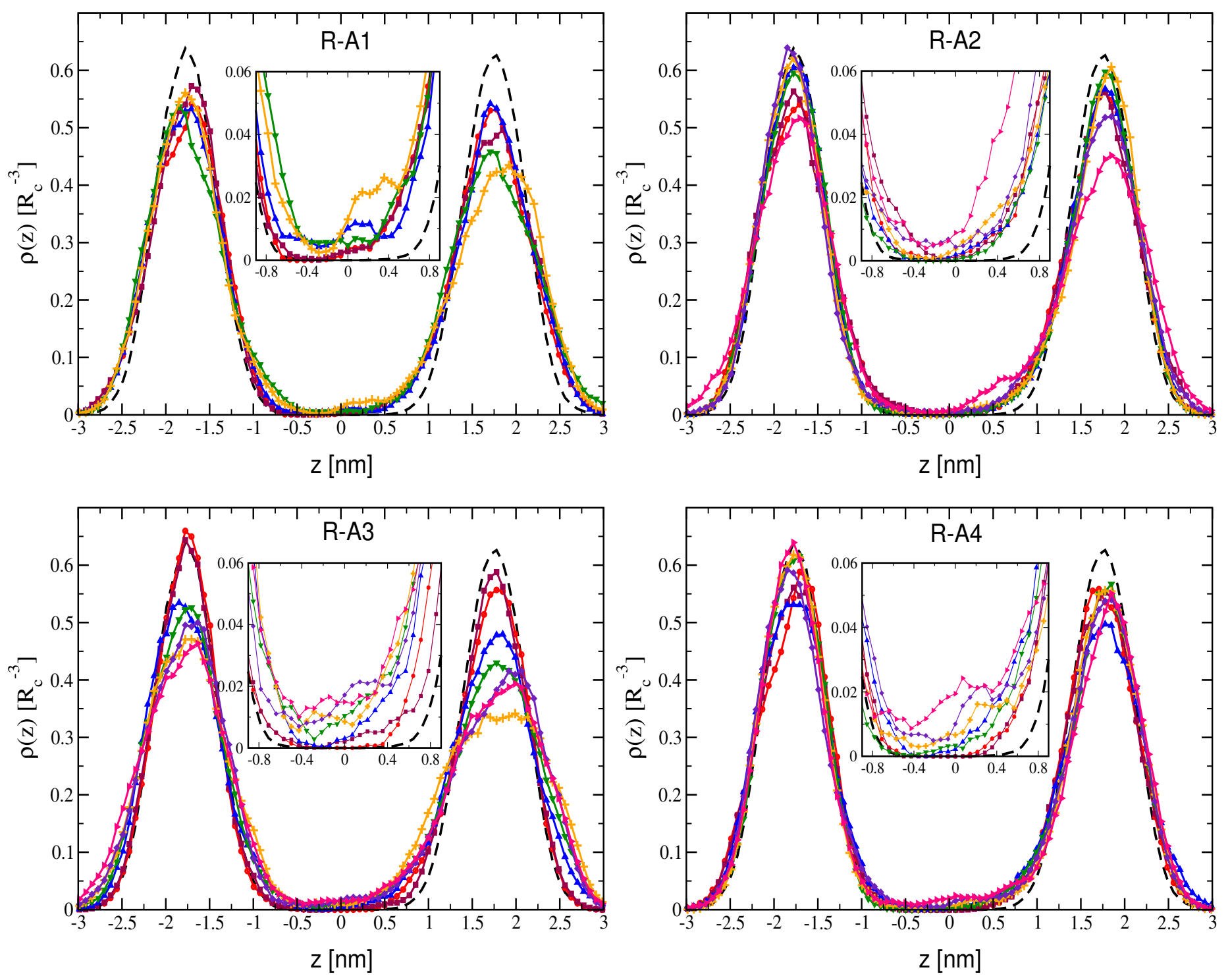

SI. Figure 7 Transient change in distribution of choline density for simulation runs R-A1, R-A2, R-A3 and R-A4 at $E_{z}=-1.8 k_{B} T / e R_{c}$. For comparison, dotted line shows the choline density average over $11.41 n s$ at $E_{z}=0$. The other symbols are $(\bigcirc)$ in time interval 0-2.28 $n s,(\square)$ in 2.28-4.56 $n s,(\triangle)$ in 4.56-6.84 $n s,(\nabla)$ in 6.84-9.13 $n s,(+)$ in 9.13-11.41 $n s,(\diamond)$ in 11.41-13.69 $n s$, and $(\triangleleft)$ over 13.69-15.97 $n s$. 

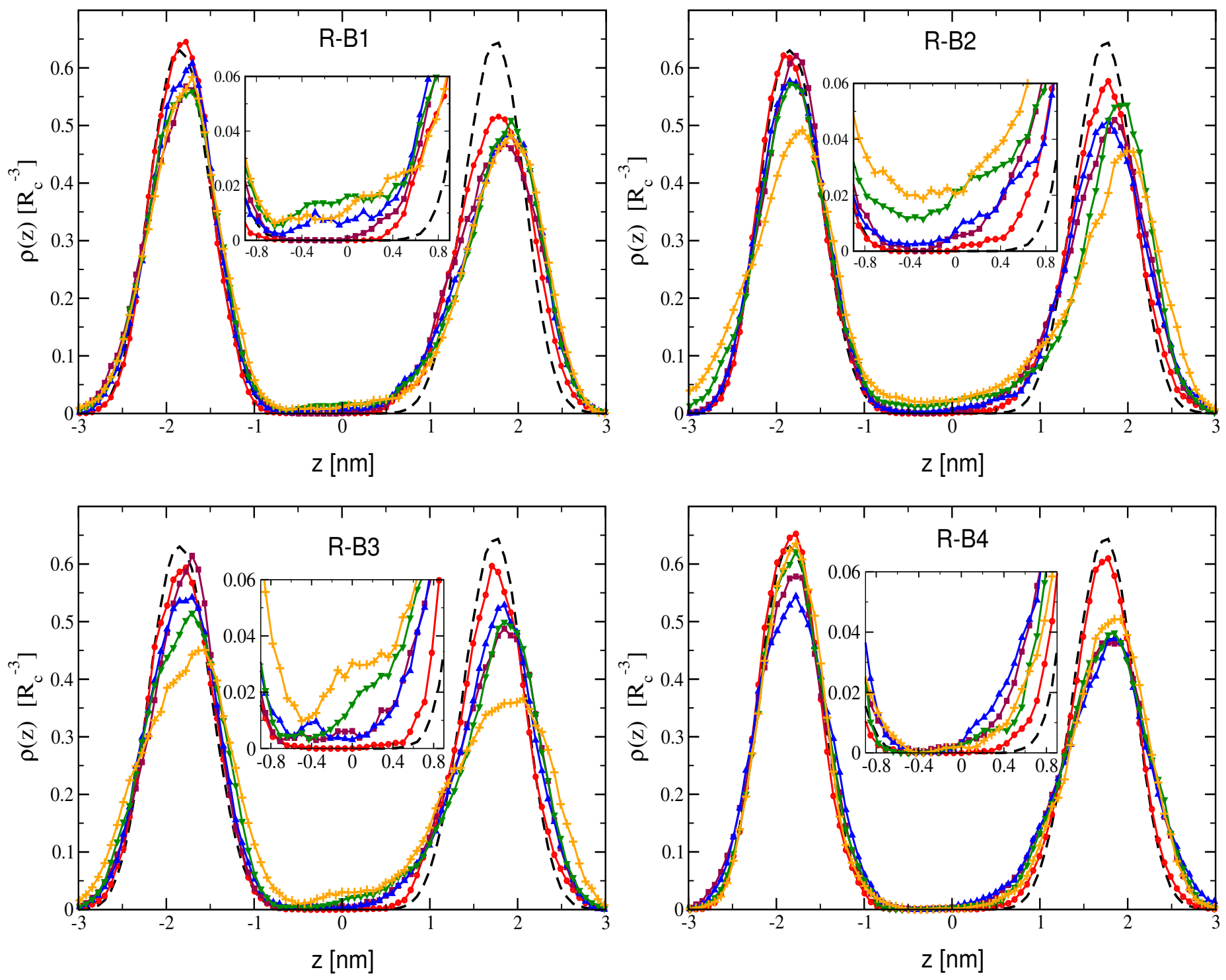

SI. Figure 8 Transient change in distribution of choline density for simulation runs R-B1, R-B2, R-B3 and R-B4 at $E_{z}=-1.4 k_{B} T / e R_{c}$. For comparison, dotted curve shows choline density averaged over $11.41 \mathrm{~ns}$ under no applied field. The other symbols are $(\bigcirc)$ in time interval 0-2.28 ns, $(\square)$ in $2.28-4.56$ $n s,(\triangle)$ in 4.56-6.84 $n s,(\nabla)$ in 6.84-9.13 $n s$, and $(+)$ over time interval 9.13-11.41 ns. 

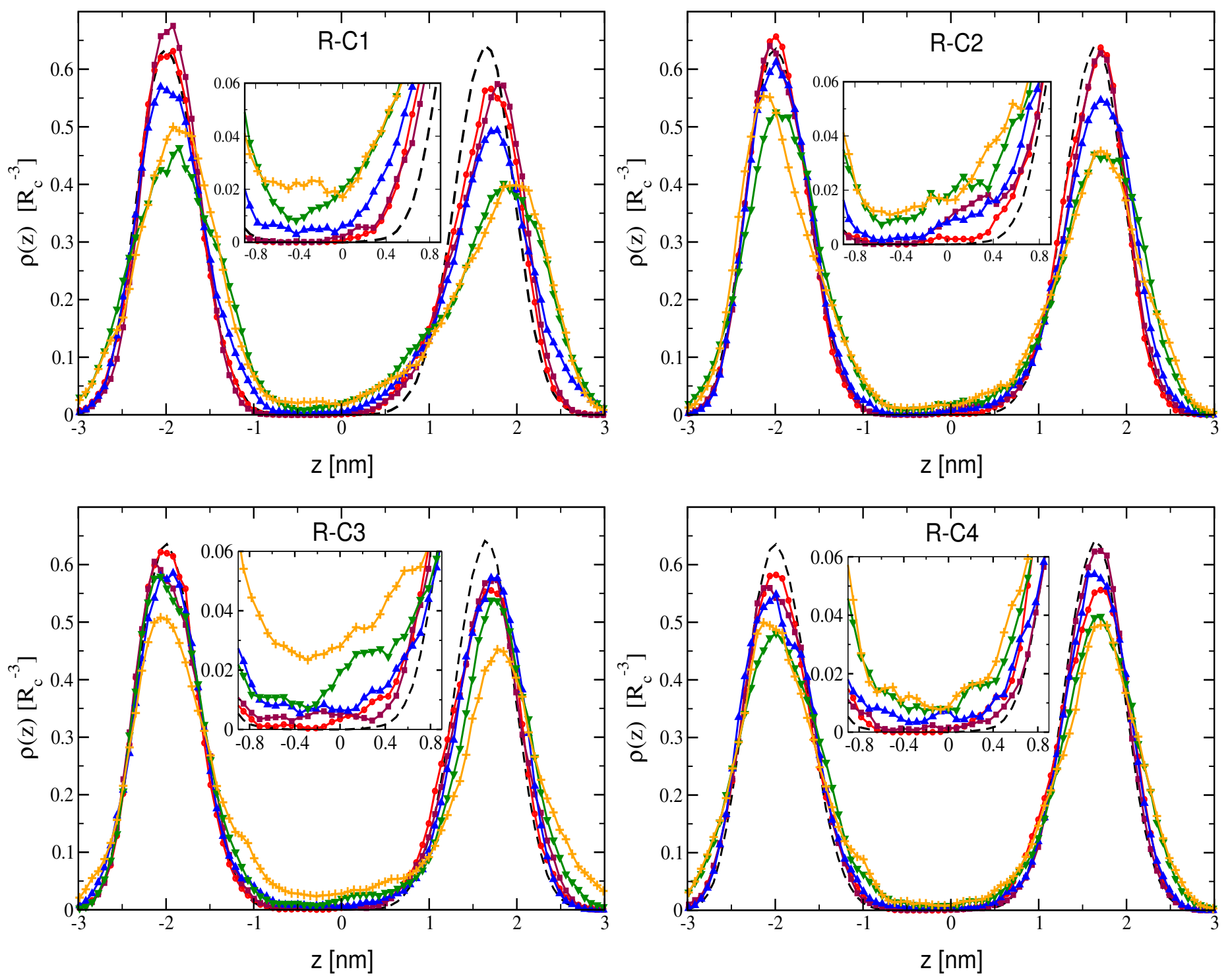

SI. Figure 9 Transient change in distribution of choline density for simulation runs R-C1, R-C2, R-C3 and R-C4 at $E_{z}=-1.4 k_{B} T / e R_{c}$. For comparison, dotted curve shows choline density averaged over $11.41 \mathrm{~ns}$ under no applied field. The other symbols are $(\bigcirc)$ in time interval 0-2.28 $\mathrm{ns}$, ( $\square)$ in $2.28-4.56$ $n s,(\triangle)$ in 4.56-6.84 $n s,(\nabla)$ in 6.84-9.13 $n s$, and $(+)$ over time interval 9.13-11.41 $n s$. 


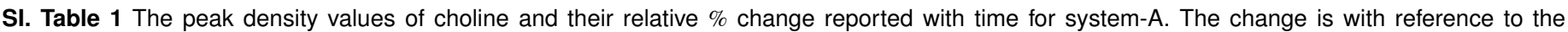

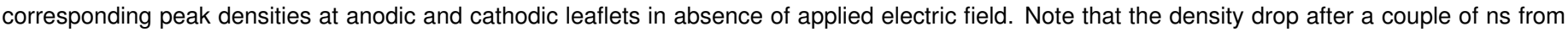
the hydrophobic pore formation is significant.

\begin{tabular}{|c|c|c|}
\hline \multirow{3}{*}{$\begin{array}{l}\text { time } \\
\text { (ns) }\end{array}$} & \multicolumn{2}{|c|}{ Density of choline in run R-A1 } \\
\hline & Anodic leaflet $\left(\% \Delta^{1}\right)$ & Cathodic leaflet $\left(\% \Delta^{1}\right)$ \\
\hline & {$\left[0.625 R_{c}^{-3}\right.$ at $\left.\mathrm{E}=0\right]$} & {$\left[0.638 R_{c}^{-3}\right.$ at $\left.\mathrm{E}=0\right]$} \\
\hline $0-2.28$ & $0.517(-17.4)$ & $0.519(-18.6)$ \\
\hline $2.28-4.56$ & $0.491(-21.5)$ & $0.555(-13.0)$ \\
\hline $4.56-6.84$ & $0.521(-16.7)$ & $0.526(-17.4)$ \\
\hline $6.84-9.13$ & $0.451(-27.9)$ & $0.512(-19.8)$ \\
\hline \multirow{2}{*}{$9.13-11.41$} & $0.438(-30.0)$ & $0.550(-13.8)$ \\
\hline & \multicolumn{2}{|c|}{ Density of choline in run R-A2 } \\
\hline $0-2.28$ & $0.539(-13.8)$ & $0.531(-16.7)$ \\
\hline $2.28-4.56$ & $0.533(-14.8)$ & $0.544(-14.7)$ \\
\hline $4.56-6.84$ & $0.543(-13.1)$ & $0.598(-6.3)$ \\
\hline $6.84-9.13$ & $0.563(-10.0)$ & $0.582(-8.8)$ \\
\hline $9.13-11.41$ & $0.573(-8.3)$ & $0.596(-6.6)$ \\
\hline $11.41-13.69$ & $0.512(-18.1)$ & $0.612(-4.1)$ \\
\hline \multirow[t]{2}{*}{$13.69-15.97$} & $0.444(-29.0)$ & $0.510(-20.1)$ \\
\hline & \multicolumn{2}{|c|}{ Density of choline in run R-A3 } \\
\hline $0-2.28$ & $0.544(-13.0)$ & $0.633(-0.7)$ \\
\hline $2.28-4.56$ & $0.562(-10.2)$ & $0.617(-3.3)$ \\
\hline $4.56-6.84$ & $0.475(-24.0)$ & $0.525(-17.6)$ \\
\hline $6.84-9.13$ & $0.435(-30.4)$ & $0.519(-18.7)$ \\
\hline $9.13-11.41$ & $0.364(-41.8)$ & $0.476(-25.4)$ \\
\hline $11.41-13.69$ & $0.418(-33.2)$ & $0.497(-22.1)$ \\
\hline \multirow[t]{2}{*}{$13.69-15.97$} & $0.400(-36.1)$ & $0.455(-28.6)$ \\
\hline & \multicolumn{2}{|c|}{ Density of choline in run R-A4 } \\
\hline $0-2.28$ & $0.545(-12.8)$ & $0.551(-13.6)$ \\
\hline $2.28-4.56$ & $0.539(-13.8)$ & $0.552(-13.5)$ \\
\hline $4.56-6.84$ & $0.492(-21.3)$ & $0.537(-15.8)$ \\
\hline $6.84-9.13$ & $0.536(-14.3)$ & $0.601(-5.7)$ \\
\hline $9.13-11.41$ & $0.537(-14.1)$ & $0.604(-5.2)$ \\
\hline $11.41-13.69$ & $0.526(-15.8)$ & $0.567(-11.0)$ \\
\hline $13.69-15.97$ & $0.521(-16.8)$ & $0.611(-4.2)$ \\
\hline
\end{tabular}

\footnotetext{
${ }^{1}$ relative $\%$ change, with standard error within $\sim 0.5$.
} 
SI. Table 2 The peak density values of choline and their relative \% change reported with time for systems-B. The change is with reference to the corresponding peak densities at anodic and cathodic leaflets in absence of applied electric field.

\begin{tabular}{|c|c|c|}
\hline \multirow{3}{*}{$\begin{array}{l}\text { time } \\
\text { (ns) }\end{array}$} & \multicolumn{2}{|c|}{ Density of choline in run R-B1 } \\
\hline & Anodic leaflet $\left(\% \Delta^{1}\right)$ & Cathodic leaflet $\left(\% \Delta^{1}\right)$ \\
\hline & {$\left[0.643 R_{c}^{-3}\right.$ at $\left.\mathrm{E}=0\right]$} & {$\left[0.629 R_{c}^{-3}\right.$ at $\left.\mathrm{E}=0\right]$} \\
\hline $0-2.28$ & $0.506(-21.2)$ & $0.625(-0.6)$ \\
\hline $2.28-4.56$ & $0.463(-27.9)$ & $0.555(-11.8)$ \\
\hline $4.56-6.84$ & $0.485(-24.6)$ & $0.589(-6.4)$ \\
\hline $6.84-9.13$ & $0.497(-22.7)$ & $0.556(-11.6)$ \\
\hline \multirow[t]{2}{*}{$9.13-11.41$} & $0.479(-25.4)$ & $0.562(-10.6)$ \\
\hline & \multicolumn{2}{|c|}{ Density of choline in run R-B2 } \\
\hline $0-2.28$ & $0.550(-14.5)$ & $0.597(-5.1)$ \\
\hline $2.28-4.56$ & $0.493(-23.3)$ & $0.602(-4.3)$ \\
\hline $4.56-6.84$ & $0.504(-21.6)$ & $0.568(-9.7)$ \\
\hline $6.84-9.13$ & $0.524(-18.5)$ & $0.561(-10.8)$ \\
\hline \multirow[t]{2}{*}{$9.13-11.41$} & $0.453(-29.5)$ & $0.487(-22.6)$ \\
\hline & \multicolumn{2}{|c|}{ Density of choline in run R-B3 } \\
\hline $0-2.28$ & $0.565(-12.2)$ & $0.578(-8.1)$ \\
\hline $2.28-4.56$ & $0.487(-24.2)$ & $0.589(-6.3)$ \\
\hline $4.56-6.84$ & $0.512(-20.4)$ & $0.540(-14.1)$ \\
\hline $6.84-9.13$ & $0.489(-24.0)$ & $0.506(-19.6)$ \\
\hline \multirow[t]{2}{*}{$9.13-11.41$} & $0.373(-41.9)$ & $0.443(-29.6)$ \\
\hline & \multicolumn{2}{|c|}{ Density of choline in run R-B4 } \\
\hline $0-2.28$ & $0.589(-8.3)$ & $0.628(-0.2)$ \\
\hline $2.28-4.56$ & $0.473(-26.5)$ & $0.569(-9.4)$ \\
\hline $4.56-6.84$ & $0.460(-28.4)$ & $0.534(-15.2)$ \\
\hline $6.84-9.13$ & $0.472(-26.6)$ & $0.595(-5.4)$ \\
\hline $9.13-11.41$ & $0.505(-21.4)$ & $0.609(-3.2)$ \\
\hline
\end{tabular}

${ }^{1}$ relative $\%$ change, within standard error $\sim 0.5$.

SI. Table 3 The peak density values of choline and their relative \% change reported with time for system $\mathrm{C}$. The change is with reference to the corresponding peak densities at anodic and cathodic leaflets in absence of applied electric field.

\begin{tabular}{|c|c|c|}
\hline \multirow{3}{*}{$\begin{array}{l}\text { time } \\
\text { (ns) }\end{array}$} & \multicolumn{2}{|c|}{ Density of choline in run R-C1 } \\
\hline & Anodic leaflet $\left(\% \Delta^{1}\right)$ & Cathodic leaflet $\left(\% \Delta^{1}\right)$ \\
\hline & {$\left[0.640 R_{c}^{-3}\right.$ at $\left.\mathrm{E}=0\right]$} & {$\left[0.635 R_{c}^{-3}\right.$ at $\left.\mathrm{E}=0\right]$} \\
\hline $0-2.28$ & $0.555(-13.3)$ & $0.619(-2.5)$ \\
\hline $2.28-4.56$ & $0.546(-14.7)$ & $0.654(3.0)$ \\
\hline $4.56-6.84$ & $0.483(-24.5)$ & $0.563(-11.3)$ \\
\hline $6.84-9.13$ & $0.403(-37.0)$ & $0.453(-28.7)$ \\
\hline \multirow[t]{2}{*}{$9.13-11.41$} & $0.408(-36.2)$ & $0.495(-22.0)$ \\
\hline & \multicolumn{2}{|c|}{ Density of choline in run R-C2 } \\
\hline $0-2.28$ & $0.610(-4.7)$ & $0.637(0.3)$ \\
\hline $2.28-4.56$ & $0.607(-5.2)$ & $0.621(-2.2)$ \\
\hline $4.56-6.84$ & $0.543(-15.2)$ & $0.596(-6.1)$ \\
\hline $6.84-9.13$ & $0.469(-24.7)$ & $0.529(-16.7)$ \\
\hline \multirow[t]{2}{*}{$9.13-11.41$} & $0.458(-28.4)$ & $0.535(-15.7)$ \\
\hline & \multicolumn{2}{|c|}{ Density of choline in run R-C3 } \\
\hline $0-2.28$ & $0.549(-14.2)$ & $0.615(-3.1)$ \\
\hline $2.28-4.56$ & $0.563(-12.0)$ & $0.582(-8.3)$ \\
\hline $4.56-6.84$ & $0.559(-12.7)$ & $0.576(-9.2)$ \\
\hline $6.84-9.13$ & $0.518(-19.1)$ & $0.566(-10.8)$ \\
\hline \multirow[t]{2}{*}{$9.13-11.41$} & $0.444(-30.6)$ & $0.504(-20.6)$ \\
\hline & \multicolumn{2}{|c|}{ Density of choline in run R-C4 } \\
\hline $0-2.28$ & $0.550(-14.1)$ & $0.576(-9.2)$ \\
\hline $2.28-4.56$ & $0.615(-3.9)$ & $0.554(-12.7)$ \\
\hline $4.56-6.84$ & $0.576(-9.9)$ & $0.536(-15.6)$ \\
\hline $6.84-9.13$ & $0.505(-21.2)$ & $0.477(-24.8)$ \\
\hline $9.13-11.41$ & $0.489(-23.5)$ & $0.494(-22.2)$ \\
\hline
\end{tabular}

\footnotetext{
${ }^{1}$ relative $\%$ change, within standard error $\sim 0.5$.
} 

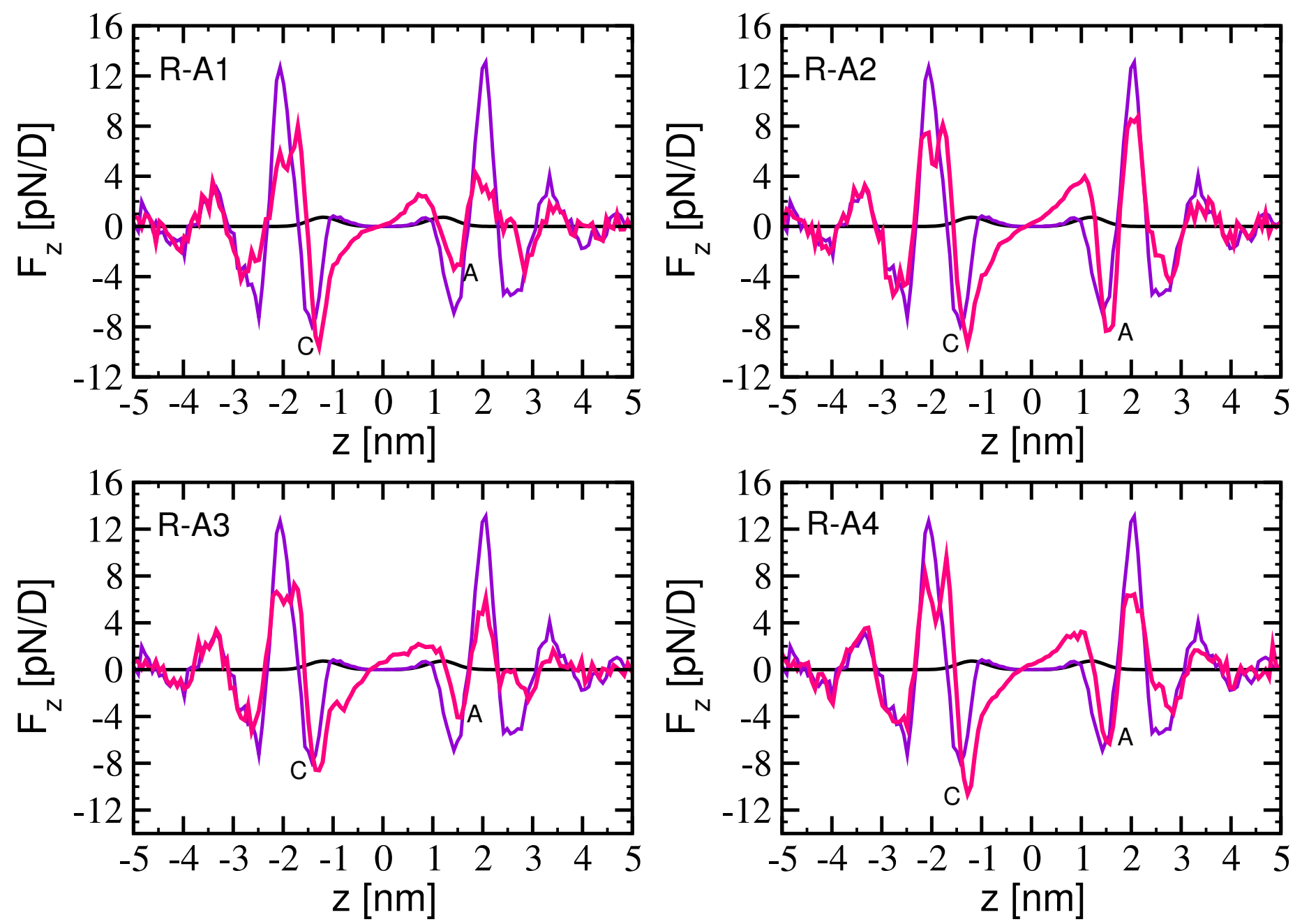

SI. Figure 10 The dielectrophoretic force on a unit dipole along the field direction for simulation runs R-A1, R-A2, R-A3 and R-A4. The colors refer to the force per unit dipole moment along z-direction under no external fields (violet), and at poration field $E_{z}=-1.8 k_{B} T / e R_{c}$ (pink). The black line indicates the density distribution of carbonyl-2 beads just to show precisely the locations of water-carbonyl interfaces. The profiles are averaged over the simulation run times. 

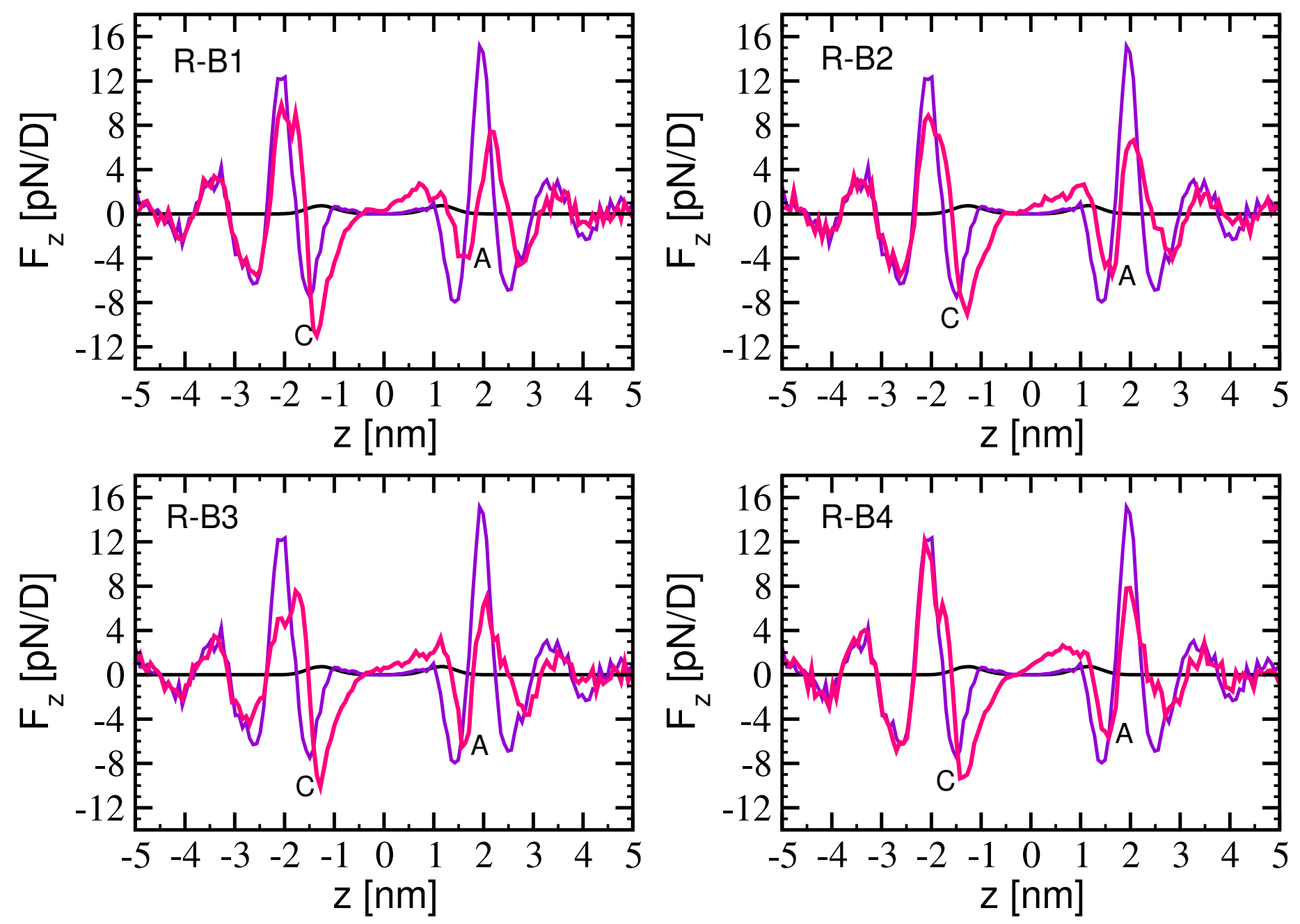

SI. Figure 11 The dielectrophoretic force on a unit dipole along the field direction for simulation runs R-B1, R-B2, R-B3 and R-B4. The colors refer to the force per unit dipole moment along z-direction under no external fields (violet), and at poration field $E_{z}=-1.4 k_{B} T / e R_{c}$ (pink). The black line indicates the density distribution of carbonyl-2 beads just to show precisely the locations of water-carbonyl interfaces. The profiles are averaged over the simulation run times. 

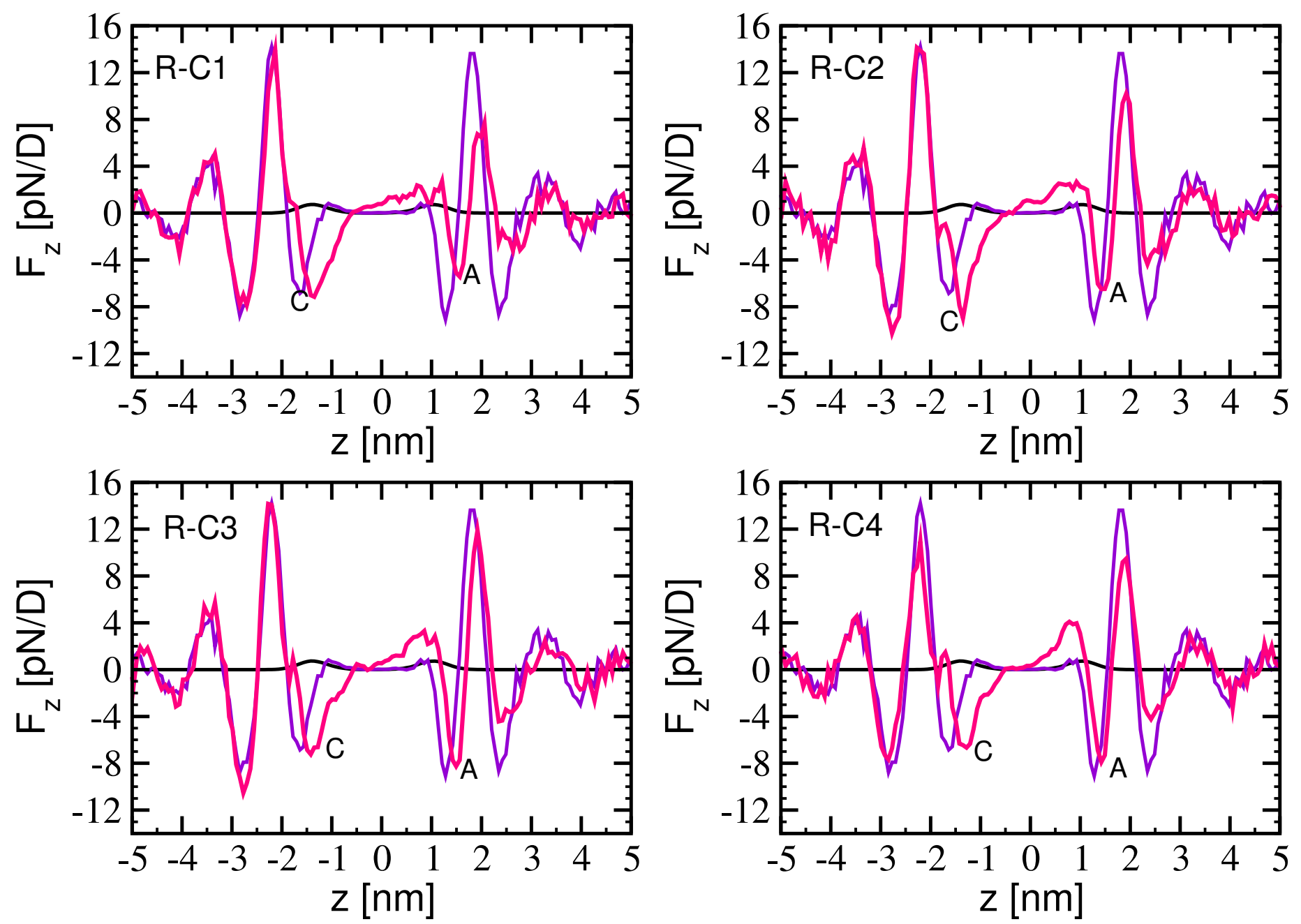

SI. Figure 12 The dielectrophoretic force on a unit dipole along the field direction for simulation runs R-C1, R-C2, R-C3 and R-C4. The colors refer to the force per unit dipole moment along z-direction under no external fields (violet), and at poration field $E_{z}=-1.4 k_{B} T / e R_{c}$ (pink). The black line indicates the density distribution of carbonyl-2 beads just to show precisely the locations of water-carbonyl interfaces. The profiles are averaged over the simulation run times. 

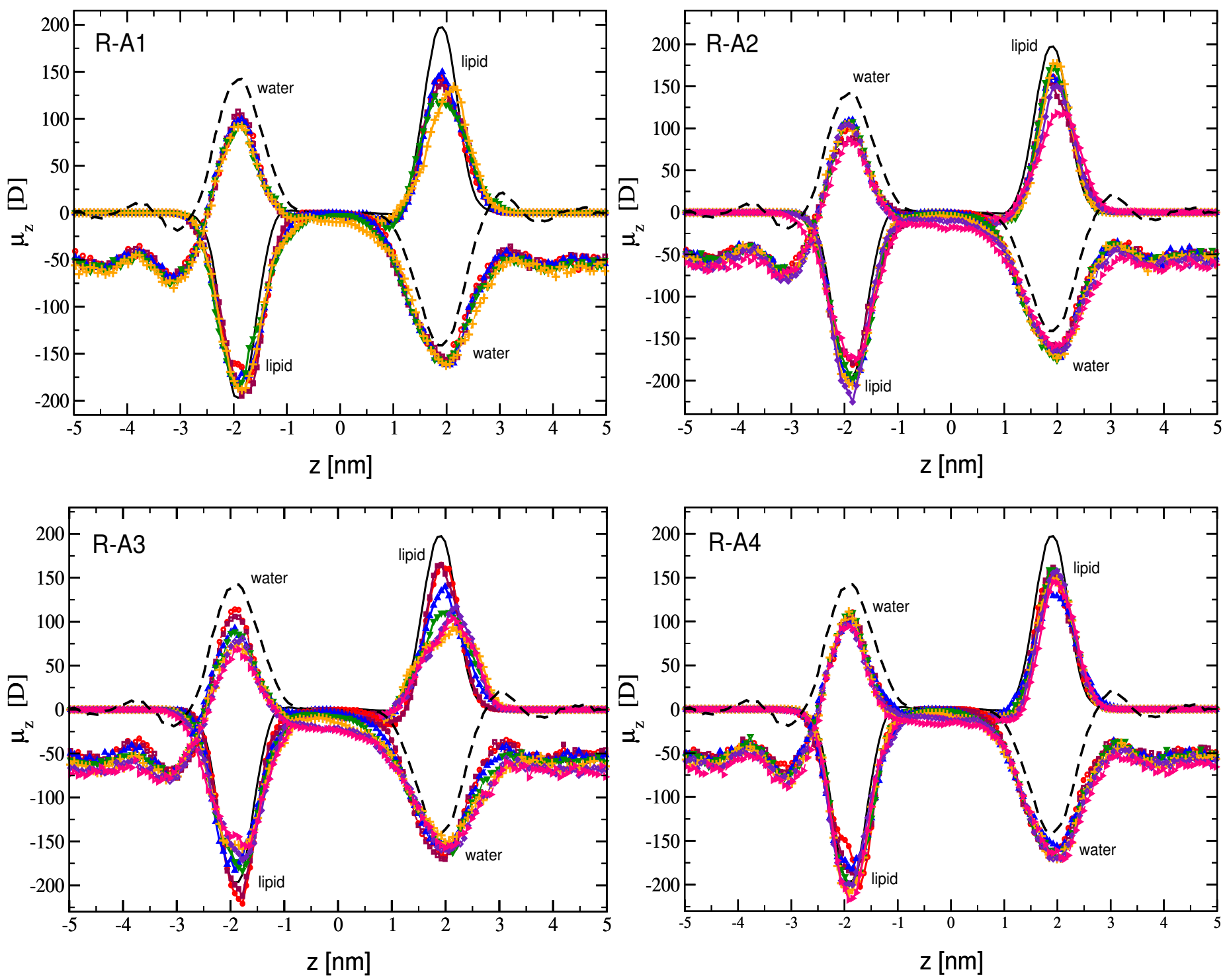

SI. Figure 13 Transient change in distribution of z-component dipole moments of lipid and water for simulation runs R-A1, R-A2, R-A3 and R-A4 at $E_{z}=-1.8 k_{B} T / e R_{c}$. For comparison, the black curves show the distributions for lipid (solid) and water (dotted), averaged over $11.41 \mathrm{~ns}$ at $E_{z}=0$. The other symbols are $(\bigcirc)$ in time interval 0-2.28 $n s,(\square)$ in 2.28-4.56 $n s,(\triangle)$ in 4.56-6.84 $n s,(\nabla)$ in 6.84-9.13 $n s,(+)$ in 9.13-11.41 ns, $(\diamond)$ in 11.41-13.69 $n s$, and $(\triangleleft)$ over 13.69-15.97 ns. 

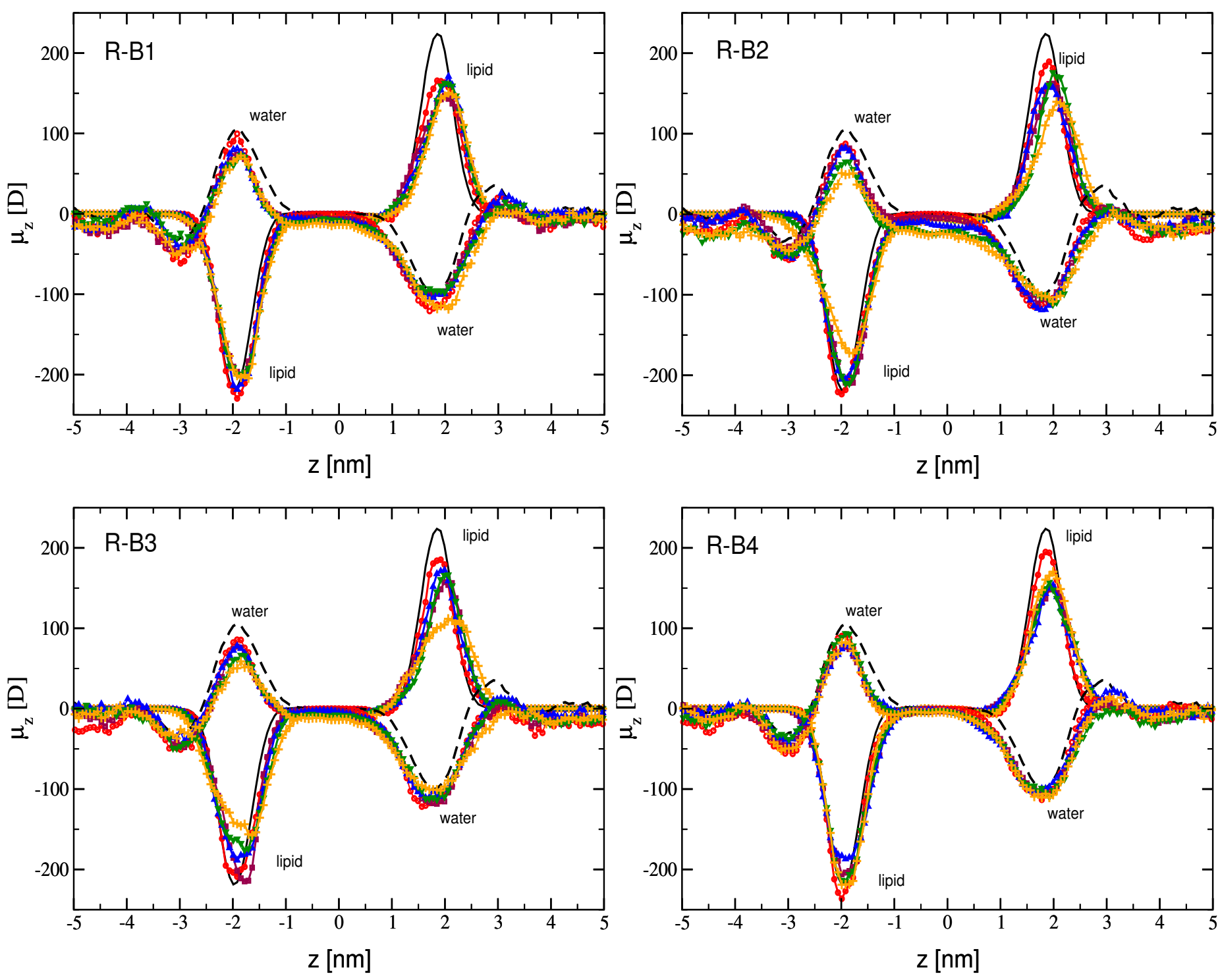

SI. Figure 14 Transient change in z-dipole moments of lipid and water for simulation runs R-B1, R-B2, R-B3 and R-B4 at $E_{z}=-1.4 k_{B} T / e R_{c}$. For comparison, the black curves show the dipoles for lipid (solid) and water (dotted), averaged over $11.41 \mathrm{~ns}$ under no applied field. The other symbols are $(\bigcirc)$ in time interval 0-2.28 $n s,(\square)$ in 2.28-4.56 $n s,(\triangle)$ in 4.56-6.84 $n s,(\nabla)$ in 6.84-9.13 $n s$, and $(+)$ in time interval 9.13-11.41 $n s$. 

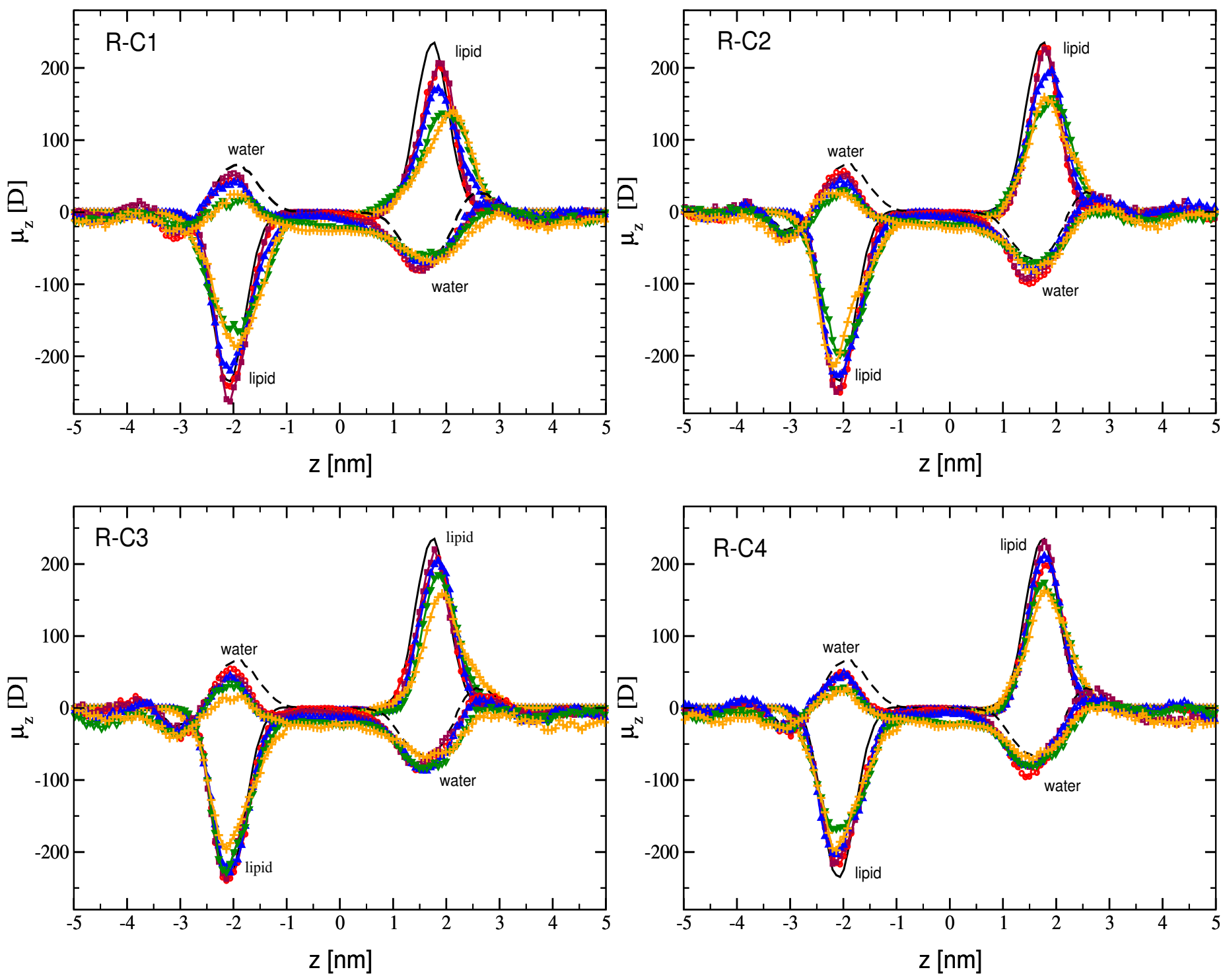

SI. Figure 15 Transient change in z-dipole moments of lipid and water for simulation runs R-C1, R-C2, R-C3 and R-C4 at $E_{z}=-1.4 k_{B} T / e R_{c}$. For comparison, the black curves show the dipoles for lipid (solid) and water (dotted), averaged over $11.41 \mathrm{~ns}$ under no applied field. The other symbols are $(\bigcirc)$ in time interval 0-2.28 $n s,(\square)$ in 2.28-4.56 $n s,(\triangle)$ in 4.56-6.84 $n s,(\nabla)$ in 6.84-9.13 $n s$, and $(+)$ in time interval 9.13-11.41 $n s$. 
SI. Table 4 The peak values of $z$-component of dipole moment of lipids, water and their relative $\%$ change reported with time for system-A. The change is with reference to the corresponding peak values at anodic and cathodic leaflets in absence of applied electric field.

\begin{tabular}{|c|c|c|c|c|}
\hline \multirow{3}{*}{$\begin{array}{l}\text { time } \\
\text { (ns) }\end{array}$} & \multicolumn{2}{|c|}{ lipid dipole moment in run R-A1 } & \multicolumn{2}{|c|}{ water dipole moment in run R-A1 } \\
\hline & $\mu_{z}$ anodic side $\left(\% \Delta_{1}^{1}\right)\left(\% \Delta_{2}^{2}\right)$ & $-\mu_{z}$ cathodic side $\left(\% \Delta_{1}^{1}\right)\left(\% \Delta_{2}^{2}\right)$ & $-\mu_{z}$ anodic side $\left(\% \Delta^{3}\right)$ & $\mu_{z}$ cathodic side $\left(\% \Delta^{3}\right)$ \\
\hline & {$[197.4 \mathrm{D}$ at $\mathrm{E}=0]$} & {$[196.7 \mathrm{D}$ at $\mathrm{E}=0]$} & {$[140.9 \mathrm{D}$ at $\mathrm{E}=0]$} & {$[142.2 \mathrm{D}$ at $\mathrm{E}=0]$} \\
\hline $0-2.28$ & $144.1(-27.0)(-9.6)$ & $177.9(-9.5)(9.1)$ & $157.7(12.0)$ & $101.8(-28.4)$ \\
\hline $2.28-4.56$ & $137.2(-30.5)(-8.9)$ & $194.5(-1.1)(11.9)$ & $156.4(11.0)$ & $107.6(-24.4)$ \\
\hline $4.56-6.84$ & $149.9(-24.0)(-7.3)$ & $181.6(-7.7)(9.8)$ & $161.8(14.8)$ & $99.5(-30.0)$ \\
\hline $6.84-9.13$ & $124.5(-36.9)(-9.0)$ & $184.3(-6.3)(13.5)$ & $159.6(13.3)$ & $92.1(-35.2)$ \\
\hline \multirow[t]{2}{*}{$9.13-11.41$} & $134.0(-32.1)(-2.1)$ & $188.3(-4.3)(9.5)$ & $162.3(15.2)$ & $92.3(-35.1)$ \\
\hline & \multicolumn{2}{|c|}{ lipid dipole moment in run R-A2 } & \multicolumn{2}{|c|}{ water dipole moment in run R-A2 } \\
\hline $0-2.28$ & $161.4(-18.2)(-4.4)$ & $181.2(-7.9)(8.9)$ & $161.4(14.6)$ & $102.7(-27.8)$ \\
\hline $2.28-4.56$ & $153.8(-22.0)(-7.3)$ & $179.0(-9.0)(5.8)$ & $162.4(15.3)$ & $88.6(-37.7)$ \\
\hline $4.56-6.84$ & $160.4(-18.7)(-5.6)$ & $202.3(2.9)(9.1)$ & $172.6(22.5)$ & $109.8(-22.8)$ \\
\hline $6.84-9.13$ & $172.6(-12.6)(-2.6)$ & $198.0(0.6)(9.4)$ & $175.7(24.7)$ & $108.0(-24.1)$ \\
\hline $9.13-11.41$ & $177.1(-10.3)(-2.0)$ & $205.5(4.5)(11.1)$ & $173.4(23.1)$ & $105.3(-26.0)$ \\
\hline $11.41-13.69$ & $150.5(-23.8)(-5.6)$ & $225.5(14.6)(18.7)$ & $164.9(17.0)$ & $104.5(-26.5)$ \\
\hline \multirow[t]{2}{*}{$13.69-15.97$} & $118.3(-40.0)(-11.0)$ & $174.66(-11.2)(8.9)$ & $159.6(13.3)$ & $86.8(-39.0)$ \\
\hline & \multicolumn{2}{|c|}{ lipid dipole moment in run R-A3 } & \multicolumn{2}{|c|}{ water dipole moment in run R-A3 } \\
\hline $0-2.28$ & $164.8(-16.5)(-3.5)$ & $220.8(12.3)(13.0)$ & $167.6(19.0)$ & $113.9(-19.9)$ \\
\hline $2.28-4.56$ & $164.6(-16.6)(-6.4)$ & $212.2(7.9)(11.1)$ & $170.2(20.8)$ & $106.0(-25.5)$ \\
\hline $4.56-6.84$ & $139.6(-29.3)(-5.2)$ & $183.0(-7.0)(10.6)$ & $155.0(10.0)$ & $92.5(-34.9)$ \\
\hline $6.84-9.13$ & $110.6(-44.0)(-13.5)$ & $183.1(-6.9)(11.7)$ & $162.6(15.4)$ & $88.6(-37.7)$ \\
\hline $9.13-11.41$ & $93.4(-52.7)(-10.9)$ & $158.4(-19.5)(5.9)$ & $151.4(7.5)$ & $74.8(-47.4)$ \\
\hline $11.41-13.69$ & $117.1(-40.7)(-7.4)$ & $171.1(-13.0)(9.1)$ & 160.5 (13.9) & $81.2(-42.9)$ \\
\hline \multirow[t]{2}{*}{$13.69-15.97$} & $104.1(-47.2)(-11.2)$ & $155.3(-21.0)(7.6)$ & $157.1(11.5)$ & $68.5(-51.8)$ \\
\hline & \multicolumn{2}{|c|}{ lipid dipole moment in run R-A4 } & \multicolumn{2}{|c|}{ water dipole moment in run R-A4 } \\
\hline $0-2.28$ & $153.9(-22.0)(-9.2)$ & $202.4(2.9)(16.5)$ & $165.9(17.8)$ & $110.5(-22.33)$ \\
\hline $2.28-4.56$ & $161.5(-18.2)(-4.4)$ & $190.3(-3.2)(10.2)$ & $164.0(16.4)$ & $108.5(-23.7)$ \\
\hline $4.56-6.84$ & $135.1(-31.6)(-10.3)$ & $182.3(-7.3)(8.5)$ & $159.9(13.5)$ & $100.2(-29.6)$ \\
\hline $6.84-9.13$ & $159.2(-19.3)(-5.0)$ & $200.7(2.0)(7.7)$ & $167.7(19.1)$ & $110.3(-22.4)$ \\
\hline $9.13-11.41$ & $149.0(-24.5)(-10.4)$ & $209.4(6.5)(11.7)$ & $170.4(21.0)$ & $111.1(-21.9)$ \\
\hline $11.41-13.69$ & $157.7(-20.1)(-4.3)$ & $200.2(1.8)(12.8)$ & $170.7(21.2)$ & $96.5(-32.2)$ \\
\hline $13.69-15.97$ & $144.9(-26.6)(-9.8)$ & $217.3(10.5)(14.7)$ & $170.7(21.2)$ & $94.7(-33.4)$ \\
\hline
\end{tabular}

${ }^{1} \Delta_{1}$ is total relative $\%$ change, within the standard error 2 ; this includes changes due to density reduction and lipid reorientation.

${ }^{2} \Delta_{2}$ is the relative $\%$ change, solely due to lipid reorientation caused by the electric field.

${ }^{3} \Delta$ is the total relative $\%$ change, within the standard error 2 . 
SI. Table 5 The peak values of z-component of dipole moment of lipids, water and their relative \% change reported with time for system B. The change is with reference to the corresponding peak values at anodic and cathodic leaflets in absence of applied electric field.

\begin{tabular}{|c|c|c|c|c|}
\hline \multirow{3}{*}{$\begin{array}{l}\text { time } \\
\text { (ns) }\end{array}$} & \multicolumn{2}{|c|}{ lipid dipole moment in run R-B1 } & \multicolumn{2}{|c|}{ water dipole moment in run R-B1 } \\
\hline & $\mu_{z}$ anodic side $\left(\% \Delta_{1}^{1}\right)\left(\% \Delta_{2}^{2}\right)$ & $-\mu_{z}$ cathodic side $\left(\% \Delta_{1}{ }^{1}\right)\left(\% \Delta_{2}^{2}\right)$ & $-\mu_{z}$ in anodic side $\left(\% \Delta^{3}\right)$ & $\mu_{z}$ in cathodic side $\left(\% \Delta^{3}\right.$ \\
\hline & {$[223.7 \mathrm{D}$ at $\mathrm{E}=0]$} & {$[218.6 \mathrm{D}$ at $\mathrm{E}=0]$} & {$[100.1 \mathrm{D}$ at $\mathrm{E}=0]$} & {$[105.6 \mathrm{D}$ at $\mathrm{E}=0]$} \\
\hline $0-2.28$ & $165.6(-26.0)(-4.7)$ & $229.6(5.0)(5.7)$ & $120.8(20.6)$ & $99.6(-5.6)$ \\
\hline $2.28-4.56$ & $146.8(-34.4)(-6.4)$ & $199.9(-8.5)(3.2)$ & $100.5(0.3)$ & $77.5(-26.5)$ \\
\hline $4.56-6.84$ & $171.1(-23.5)(1.1)$ & $218.4(-0.1)(6.2)$ & $104.4(4.2)$ & $81.1(-23.2)$ \\
\hline $6.84-9.13$ & $162.6(-27.3)(-4.6)$ & $200.1(-8.4)(3.1)$ & $97.7(-2.4)$ & $73.1(-30.8)$ \\
\hline \multirow[t]{2}{*}{$9.13-11.41$} & $150.6(-32.7)(-7.3)$ & $204.5(-6.4)(4.2)$ & $118.4(18.2)$ & $72.6(-31.3)$ \\
\hline & \multicolumn{2}{|c|}{ lipid dipole moment in run R-B2 } & \multicolumn{2}{|c|}{ water dipole moment in run R-B2 } \\
\hline $0-2.28$ & $189.5(-15.3)(-0.9)$ & $223.3(2.1)(7.2)$ & $114.5(14.4)$ & $102.7(-17.2)$ \\
\hline $2.28-4.56$ & $162.1(-27.6)(-4.2)$ & $209.6(-4.1)(0.2)$ & $110.8(10.7)$ & $88.6(-21.8)$ \\
\hline $4.56-6.84$ & $158.3(-29.2)(-7.6)$ & $204.9(-6.3)(3.4)$ & $118.8(18.6)$ & $109.8(-21.9)$ \\
\hline $6.84-9.13$ & $176.0(-21.3)(-2.8)$ & $210.2(-3.8)(7.0)$ & $112.0(11.9)$ & $108.0(-38.5)$ \\
\hline \multirow[t]{2}{*}{$9.13-11.41$} & $139.7(-37.5)(-8.0)$ & $172.7(-21.0)(1.6)$ & $108.6(8.5)$ & $105.3(-51.7)$ \\
\hline & \multicolumn{2}{|c|}{ lipid dipole moment in run R-B3 } & \multicolumn{2}{|c|}{ water dipole moment in run R-B3 } \\
\hline $0-2.28$ & $185.3(-17.2)(-5)$ & $208.9(-4.4)(3.6)$ & $121.8(21.6)$ & $85.7(-18.8)$ \\
\hline $2.28-4.56$ & $157.4(-29.6)(-5.5)$ & $214.7(-1.8)(4.5)$ & $118.3(18.1)$ & $77.2(-26.8)$ \\
\hline $4.56-6.84$ & $172.2(-23.0)(-2.6)$ & $188.5(-13.8)(0.3)$ & $110.7(10.5)$ & $77.9(-26.1)$ \\
\hline $6.84-9.13$ & $166.8(-25.4)(-1.4)$ & $175.7(-19.6)(-0.1)$ & $113.2(13.1)$ & $66.6(-36.9)$ \\
\hline \multirow[t]{2}{*}{$9.13-11.41$} & $111.8(-50.0)(-8.0)$ & $157.6(-27.9)(1.6)$ & $101.9(1.8)$ & $58.9(-44.2)$ \\
\hline & \multicolumn{2}{|c|}{ lipid dipole moment in run R-B4 } & \multicolumn{2}{|c|}{ water dipole moment in run R-B4 } \\
\hline $0-2.28$ & $194.9(-12.9)(-4.6)$ & $236.4(8.1)(8.3)$ & $113.3(13.1)$ & $92.4(-12.5)$ \\
\hline $2.28-4.56$ & $151.6(-32.2)(-5.7)$ & $206.0(-5.7)(3.7)$ & $106.3(6.1)$ & $76.9(-27.2)$ \\
\hline $4.56-6.84$ & $153.6(-31.4)(-2.9)$ & $186.9(-14.5)(0.7)$ & $104.8(4.7)$ & $80.6(-23.6)$ \\
\hline $6.84-9.13$ & $157.3(-29.7)(-3.1)$ & $214.6(-1.8)(3.6)$ & $109.8(9.6)$ & $93.5(-11.4)$ \\
\hline $9.13-11.41$ & $170.4(-23.9)(-2.5)$ & $218.9(0.1)(3.4)$ & $110.6(10.5)$ & $82.7(-21.7)$ \\
\hline
\end{tabular}

\footnotetext{
${ }^{1} \Delta_{1}$ is total relative \% change, within the standard error 2 ; this includes changes due to density reduction and lipid reorientation.

${ }^{2} \Delta_{2}$ is the relative $\%$ change, solely due to lipid reorientation caused by the applied field.

${ }^{3} \Delta$ is the total relative $\%$ change, within the standard error 2 .
} 
SI. Table 6 The peak values of z-component of dipole moment of lipids, water and their relative \% change reported with time for system C. The change is with reference to the corresponding peak values at anodic and cathodic leaflets in absence of applied electric field.

\begin{tabular}{|c|c|c|c|c|}
\hline \multirow{2}{*}{$\begin{array}{l}\text { time } \\
\text { (ns) }\end{array}$} & \multicolumn{2}{|c|}{ lipid dipole moment in run R-C1 } & \multicolumn{2}{|c|}{ water dipole moment in run R-C1 } \\
\hline & $\begin{array}{l}\mu_{z} \text { anodic side }\left(\% \Delta_{1}{ }^{1}\right)\left(\% \Delta_{2}^{2}\right) \\
{[235.0 \mathrm{D} \text { at } \mathrm{E}=0]}\end{array}$ & $\begin{array}{l}-\mu_{z} \text { cathodic side }\left(\% \Delta_{1}{ }^{1}\right)\left(\% \Delta_{2}^{2}\right) \\
{[234.6 \mathrm{D} \text { at } \mathrm{E}=0]}\end{array}$ & $\begin{array}{l}-\mu_{z} \text { in anodic side }\left(\% \Delta^{3}\right) \\
{[70.2 \mathrm{D} \text { at } \mathrm{E}=0]}\end{array}$ & $\begin{array}{l}\mu_{z} \text { in cathodic side }\left(\% \Delta^{3}\right. \\
{[66.2 \mathrm{D} \text { at } \mathrm{E}=0]}\end{array}$ \\
\hline $0-2.28$ & $203.2(-13.5)(-0.3)$ & $241.5(2.9)(5.5)$ & $80.9(15.3)$ & $46.4(-29.9)$ \\
\hline $2.28-4.56$ & $206.9(-12.0)(2.7)$ & $262.6(11.9)(8.9)$ & $81.2(15.7)$ & $54.9(-17.1)$ \\
\hline $4.56-6.84$ & $171.3(-27.1)(-2.6)$ & $220.1(-6.2)(5.2)$ & $70.0(-0.2)$ & $41.8(-36.9)$ \\
\hline $6.84-9.13$ & $137.3(-41.6)(-4.5)$ & $166.7(-28.9)(-0.3)$ & $60.1(-14.3)$ & $18.6(-71.9)$ \\
\hline \multirow[t]{2}{*}{$9.13-11.41$} & $141.1(-40.0)(-3.7)$ & $187.0(-20.3)(1.7)$ & $69.7(-0.7)$ & $24.8(-62.6)$ \\
\hline & \multicolumn{2}{|c|}{ lipid dipole moment in run $\mathrm{R}-\mathrm{C} 2$} & \multicolumn{2}{|c|}{ water dipole moment in run R-C2 } \\
\hline $0-2.28$ & $228.4(-2.8)(1.9)$ & $250.9(7.0)(6.6)$ & $100.0(42.5)$ & $56.9(-14.1)$ \\
\hline $2.28-4.56$ & $225.6(-4.0)(1.1)$ & $250.2(6.7)(8.8)$ & $93.0(32.5)$ & $50.4(-23.9)$ \\
\hline $4.56-6.84$ & $196.0(-16.6)(-1.4)$ & $227.3(-3.1)(3.0)$ & $83.4(18.8)$ & $43.8(-33.8)$ \\
\hline $6.84-9.13$ & $158.3(-32.6)(-7.9)$ & $198.2(-15.5)(1.2)$ & $72.2(2.9)$ & $30.6(-53.8)$ \\
\hline \multirow[t]{2}{*}{$9.13-11.41$} & $159.1(-32.3)(-3.9)$ & $214.8(-8.4)(7.3)$ & $84.9(21.0)$ & $26.0(-60.7)$ \\
\hline & \multicolumn{2}{|c|}{ lipid dipole moment in run R-C3 } & \multicolumn{2}{|c|}{ water dipole moment in run R-C3 } \\
\hline $0-2.28$ & $208.3(-11.4)(2.3)$ & $239.8(2.2)(5.3)$ & $86.0(22.6)$ & $54.5(-17.6)$ \\
\hline $2.28-4.56$ & $220.4(-6.2)(5.8)$ & $237.4(1.2)(9.5)$ & $80.0(14.5)$ & $43.1(-34.8)$ \\
\hline $4.56-6.84$ & $203.9(-13.2)(-0.5)$ & $228.8(-2.5)(6.7)$ & $87.3(24.4)$ & $44.8(-32.3)$ \\
\hline $6.84-9.13$ & $185.0(-21.3)(-2.2)$ & $227.6(-3.0)(7.9)$ & $83.2(18.6)$ & $34.3(-48.1)$ \\
\hline \multirow[t]{2}{*}{$9.13-11.41$} & $158.7(-32.5)(-1.8)$ & $195.8(-16.5)(4.1)$ & $69.1(-1.5)$ & $16.5(-75.0)$ \\
\hline & \multicolumn{2}{|c|}{ lipid dipole moment in run R-C4 } & \multicolumn{2}{|c|}{ water dipole moment in run R-C4 } \\
\hline $0-2.28$ & $198.4(-15.6)(-1.5)$ & $216.9(-7.5)(1.7)$ & $95.8(36.5)$ & $49.7(-24.9)$ \\
\hline $2.28-4.56$ & $230.9(-1.8)(2.2)$ & $216.3(-7.8)(4.9)$ & $81.8(16.5)$ & $45.2(-31.6)$ \\
\hline $4.56-6.84$ & $211.1(-10.2)(-0.2)$ & $203.5(-13.3)(2.3)$ & $79.7(13.6)$ & $48.8(-26.3)$ \\
\hline $6.84-9.13$ & $174.5(-25.7)(-4.6)$ & $167.8(-28.5)(-3.6)$ & $79.7(13.6)$ & $28.9(-56.3)$ \\
\hline $9.13-11.41$ & $162.9(-30.7)(-7.1)$ & $197.8(-15.7)(6.6)$ & $71.9(2.5)$ & $27.5(-58.5)$ \\
\hline
\end{tabular}

\footnotetext{
${ }^{1} \Delta_{1}$ is total relative $\%$ change, within the standard error 2 ; this includes changes due to density reduction and lipid reorientation.

${ }^{2} \Delta_{2}$ is the relative $\%$ change, solely due to lipid reorientation caused by the applied field.

${ }^{3} \Delta$ is the total relative $\%$ change, within the standard error 2 .
} 


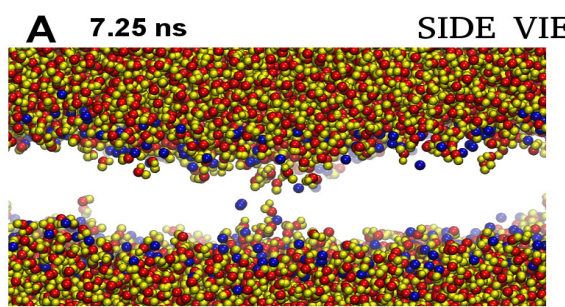

\section{B $7.76 \mathrm{~ns}$}

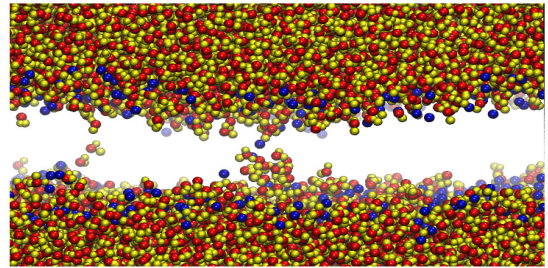

C $8.33 \mathrm{~ns}$

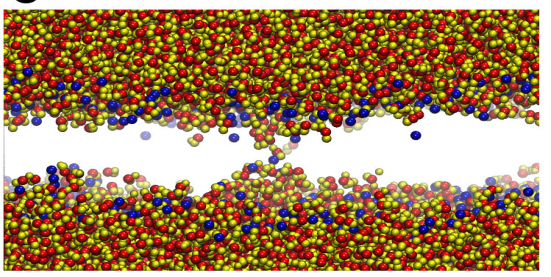

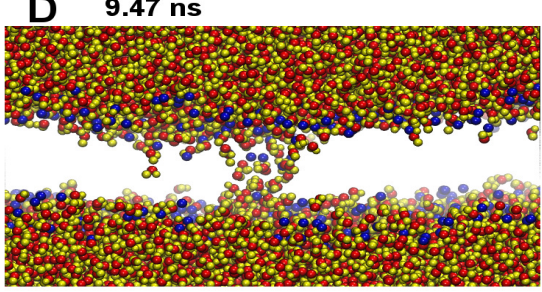

E $\quad 10.01 \mathrm{~ns}$
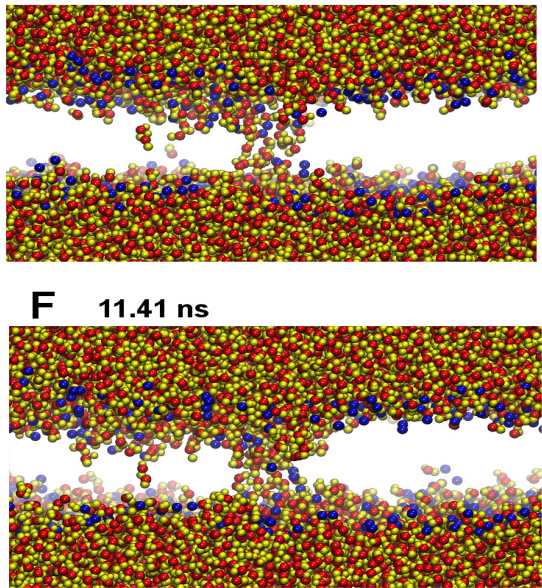
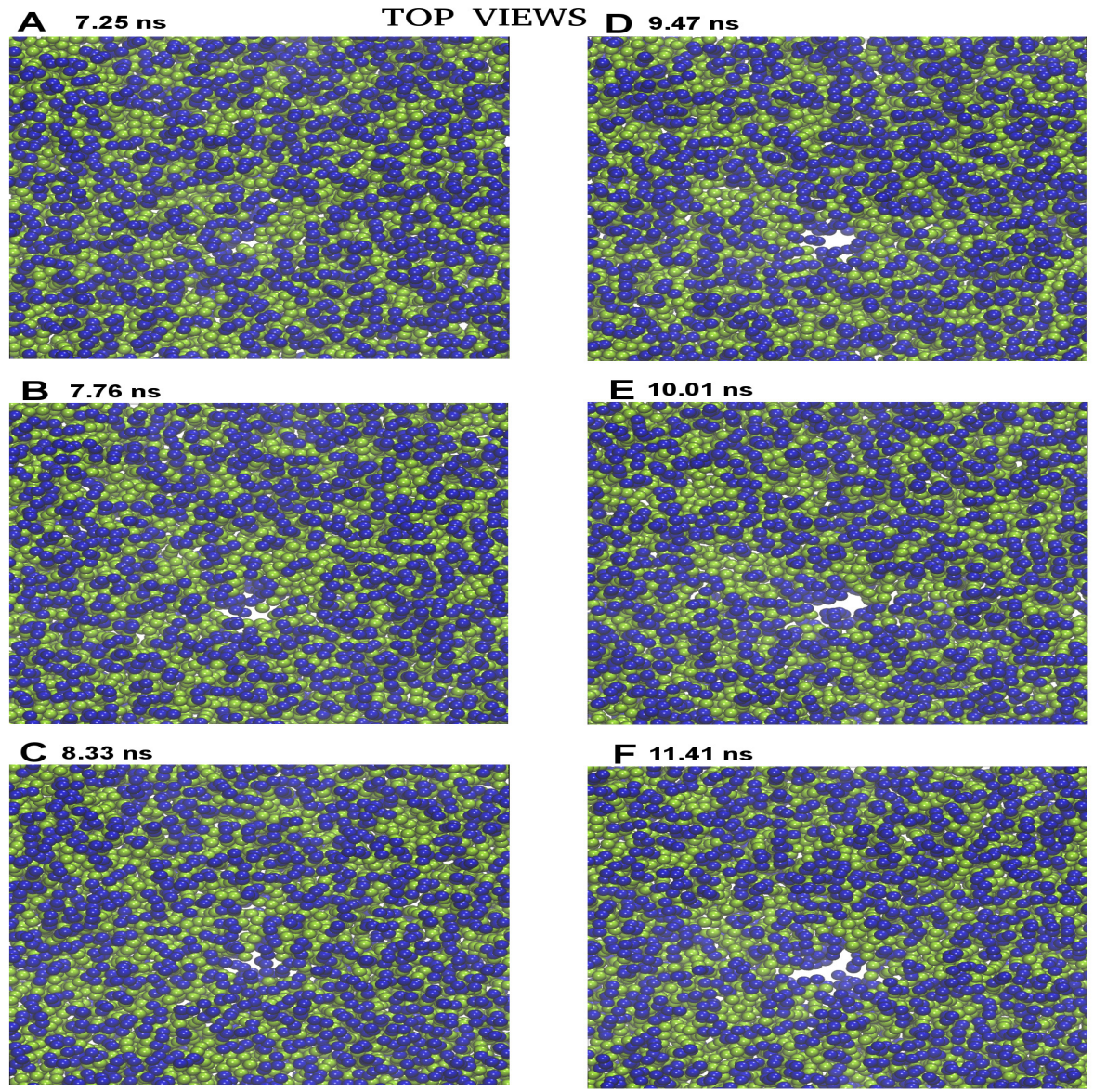

SI. Figure 16 Simulation snapshots from run R-A1, (top) side views and (bottom) top views at specified times. Water is indicated by red color with yellow Drudes. Blue and green colors respectively represent the head and tail beads of lipids. In side views, only choline and water are shown, while in top views water is not shown for clarity. 


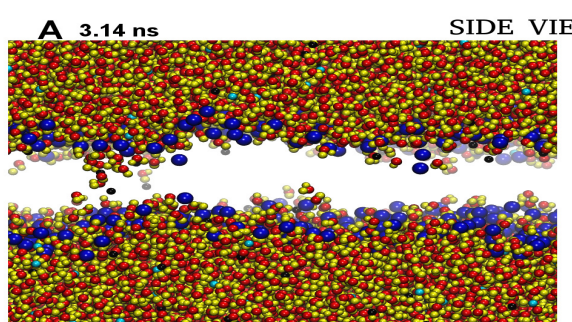

B $5.42 \mathrm{~ns}$

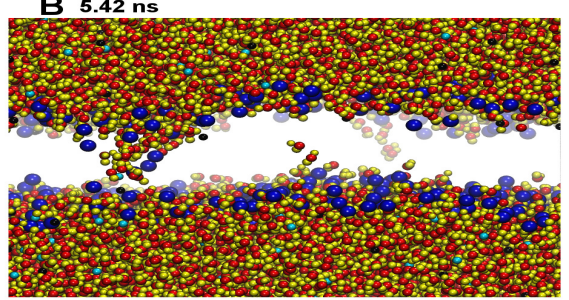

C $6.55 \mathrm{~ns}$

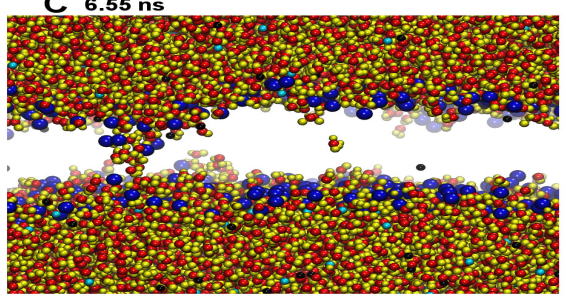

A $3.14 \mathrm{~ns}$

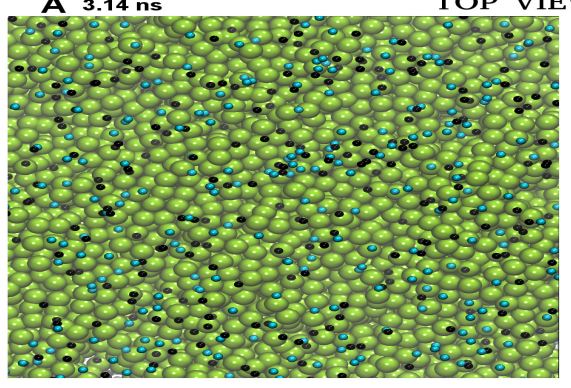

B $5.42 \mathrm{~ns}$

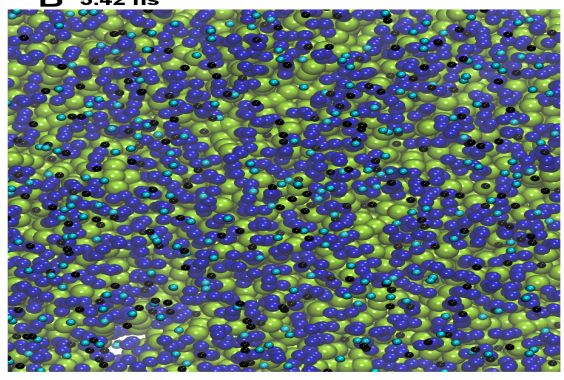

C $6.55 \mathrm{~ns}$

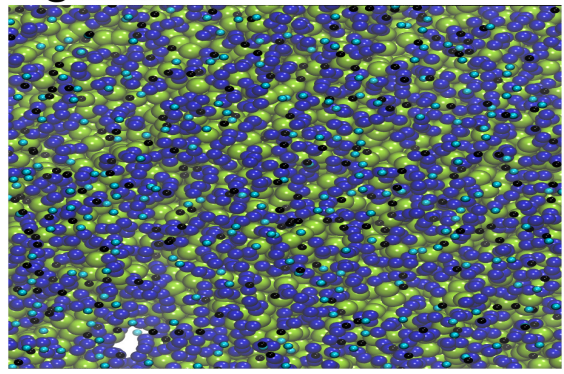

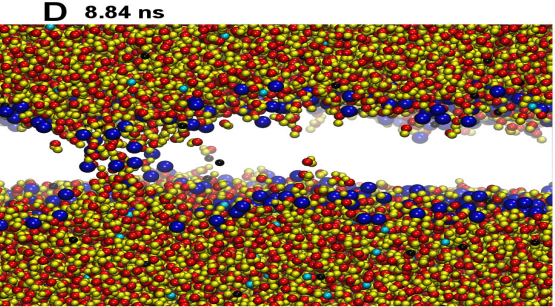

E $9.98 \mathrm{~ns}$

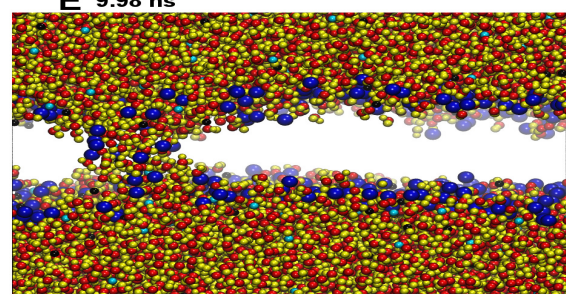

F $11.41 \mathrm{~ns}$

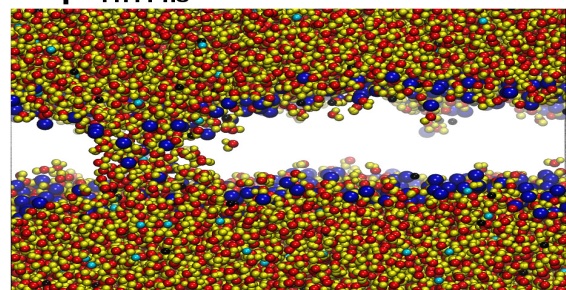

D 8.84 ns

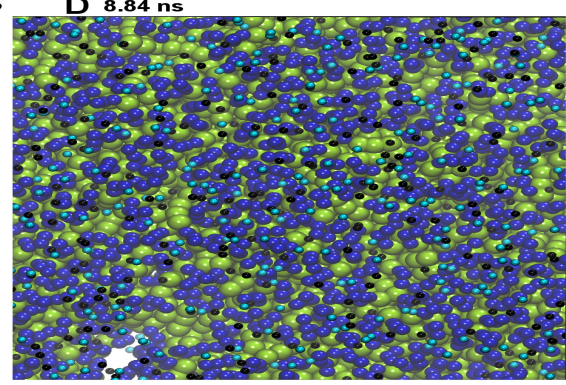

E $9.98 \mathrm{~ns}$

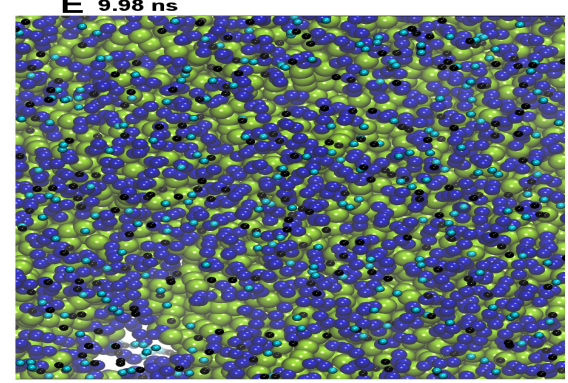

F $11.41 \mathrm{~ns}$

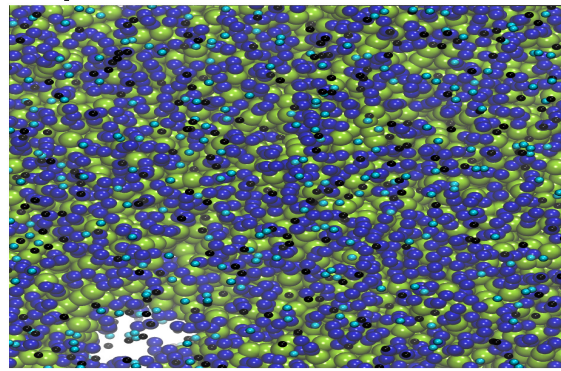

SI. Figure 17 Simulation snapshots from run R-B1, (top) side views and (bottom) top views with time at field $E_{z}=-1.4 k_{B} T / e R_{c}$. Water is indicated by red color with yellow Drudes. Blue and green colors respectively represent the head and tail beads, while black and cyan colored ions are cations and anions, respectively. In side views, only choline, water and ions are shown, while in top views water is not shown for clarity in all images, and lipids are not shown in the image-A. 

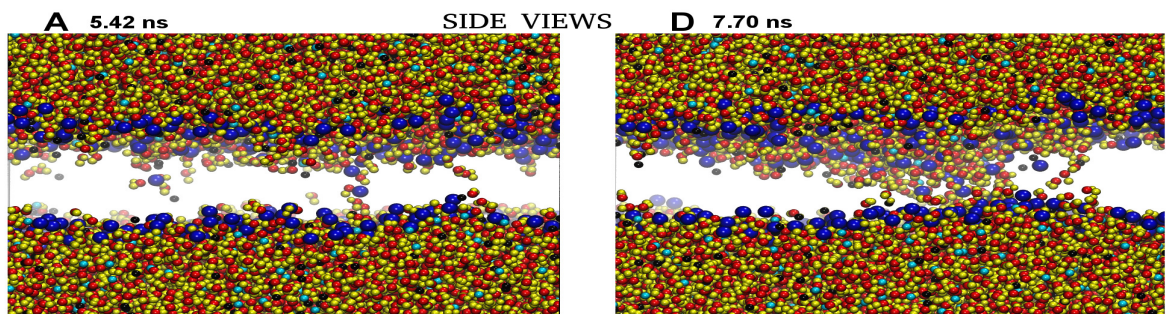

B $5.99 \mathrm{~ns}$

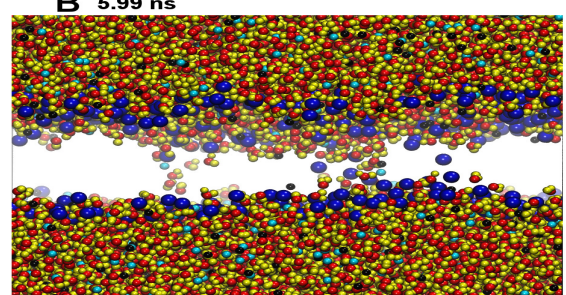

C $6.56 \mathrm{~ns}$
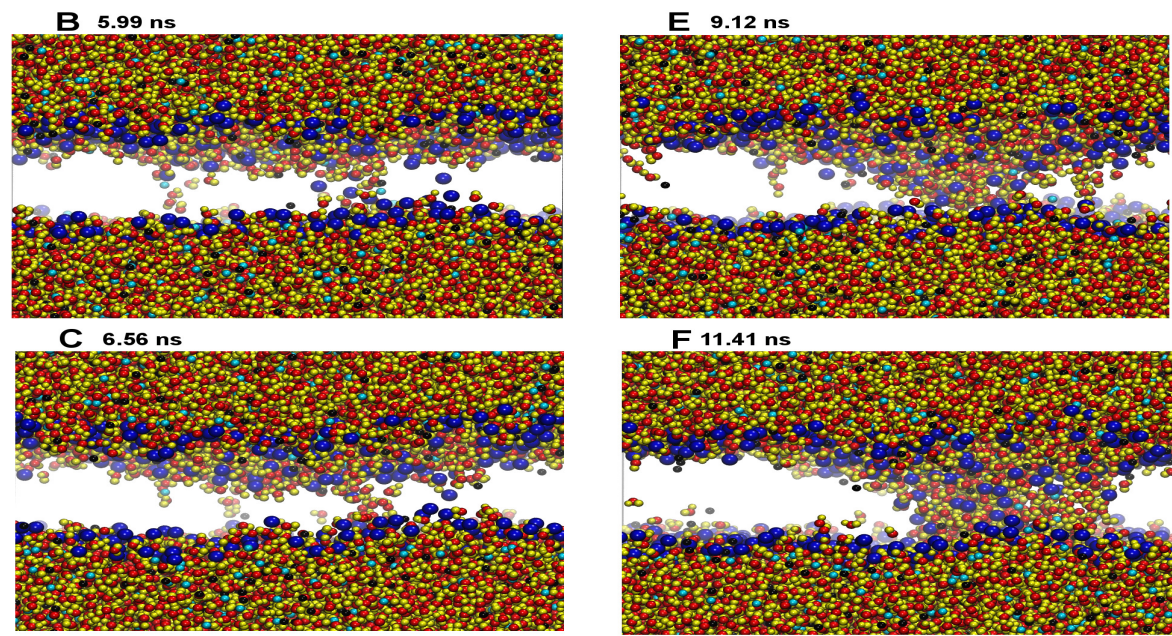

F 11.41 ns
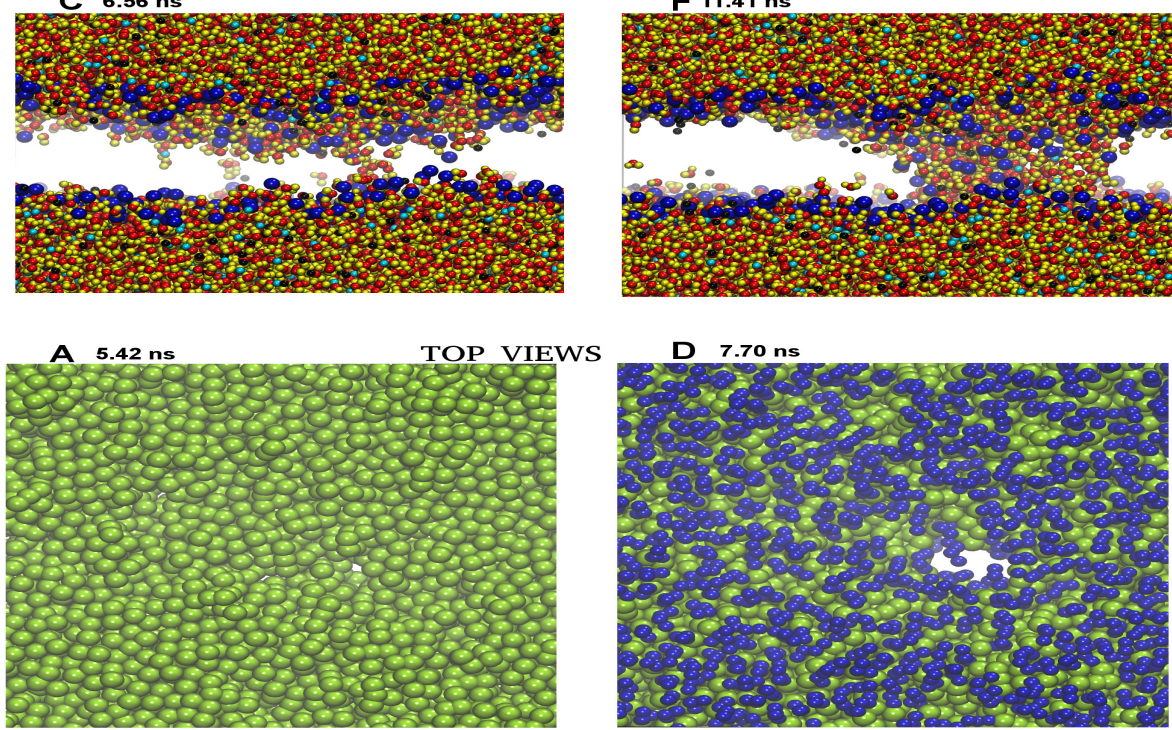

B $5.99 \mathrm{~ns}$

E $9.12 \mathrm{~ns}$

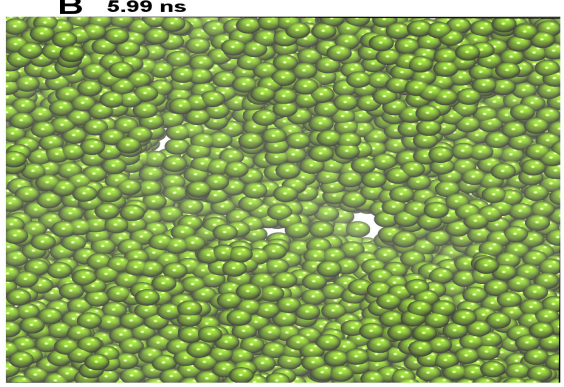

C $6.56 \mathrm{~ns}$

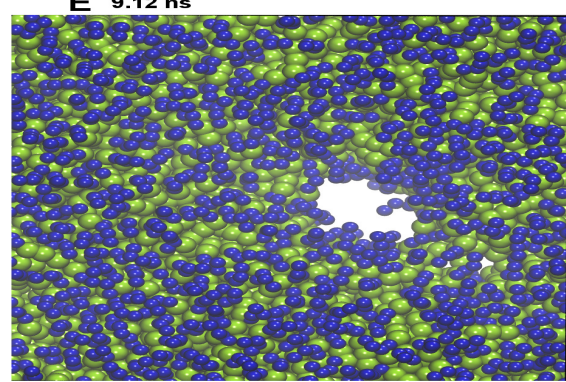

F $11.41 \mathrm{~ns}$
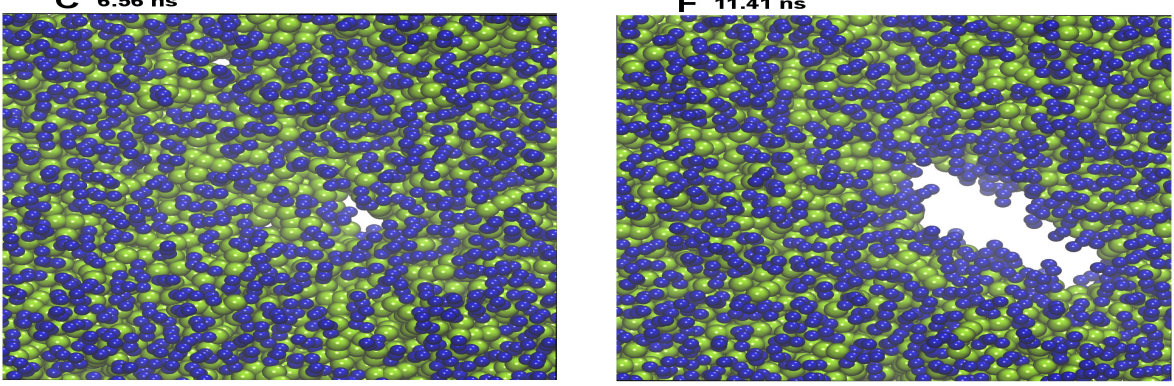

SI. Figure 18 Simulation snapshots from run R-C1, (top) side views and (bottom) top views with time at field $E_{z}=-1.4 k_{B} T / e R_{c}$. Water is indicated by red color with yellow Drudes. Blue and green colors respectively represent the head and tail beads, while black and cyan colored ions are cations and anions, respectively. In side views, only choline, water and ions are shown, while in top views water and ions are not shown for clarity in all images, and lipids are not shown in the images $A$ and $B$. 